\title{
The Initial Conditions for Protostellar Collapse: Observational Constraints
}

\author{
Philippe André \\ Service d'Astrophysique, CEA/Saclay
}

\begin{abstract}
Despite recent progress, the first stages of cloud fragmentation and core collapse remain poorly known. Significant observational advances were achieved in this field over the last decade, thanks to the use of large (sub)millimeter radiotelescopes such as the IRAM $30 \mathrm{~m}$ and the JCMT. Young protostars ('Class 0' objects), featuring powerful jets and marked infall motions, were identified at the beginning of the main accretion phase. The density structure of numerous prestellar condensations was also measured, setting strong constraints on the initial conditions for individual collapse in molecular clouds. More recently, the advent of large-format bolometer arrays has allowed complete imaging of several nearby protoclusters in the submillimeter. The results suggest that stars are generally built from finite reservoirs of mass and imply that the stellar initial mass function (IMF) is at least partly determined by turbulent fragmentation at the prestellar stage of star formation. This field will greatly benefit from future large submillimeter projects such as FIRST/Herschel and ALMA in which Europe is involved at the highest level.
\end{abstract}

\section{Introduction}

Although the formation of isolated low-mass stars is now reasonably well understood in outline (e.g. Shu, Adams, \& Lizano 1987), the very first stages of the process, which bracket in time the onset of local protostellar collapse within molecular clouds, remain poorly known, especially in star-forming clusters. These early stages are of crucial interest since they can differentiate between collapse models and, to some extent at least, must govern the origin of stellar masses. Here, after introducing the context ( $\S 1.1$ and $\S 1.2$ ), we review recent observational progress concerning the density and velocity structure of cloud cores in $\S 2$ and $\S 3$, respectively. These observations are compared with theoretical models in $\S 4$. We discuss more global results on the demography of prestellar cores in $\S 5$ and conclude in $\S 6$.

\subsection{Main conceptual phases of individual star formation}

The formation of low-mass stars is believed to involve a series of conceptually different stages (e.g. Larson 1969, Shu et al. 1987). The first stage corresponds to the (precollapse) fragmentation of a molecular cloud into a number of gravitationally-bound cores which are initially supported against gravity by a combination of thermal, magnetic, and turbulent pressures (e.g. Mouschovias 1991, Vázquez-Semadeni et al. 2000). These prestellar condensations/fragments form and evolve as a result of a still poorly understood 
mechanism, involving ambipolar diffusion (e.g. Mouschovias 1991), the dissipation of turbulence (e.g. Myers 1983, Nakano 1998), and/or an outside impulse (e.g. Bonnell et al. 1997). At some point, a given condensation becomes gravitationally unstable and quickly collapses to form a (possibly multiple) star. If we ignore fragmentation during collapse (see $\S 3.3$ below), the main theoretical features of the dynamical evolution that follows the onset of the gravitational instability have been known since the pioneering work of Larson (1969). During a probably brief initial phase, the released gravitational energy is freely radiated away and the collapsing fragment stays roughly isothermal. This "runaway" isothermal collapse phase tends to produce a strong central concentration of matter with a radial density gradient approaching $\rho \propto r^{-2}$ at small radii essentially independently of initial conditions (e.g. Whitworth \& Summers 1985, Blottiau et al. 1988, Foster \& Chevalier 1993). It ends with the formation of an opaque, hydrostatic protostellar object in the center (e.g. Larson 1969, Boss \& Yorke 1995, Bate 1998). Numerical simulations in fact predict the successive formations of two hydrostatic objects, before and after the dissociation of molecular hydrogen respectively (see Boss \& Yorke 1995, Masunaga \& Inutsuka 2000), but we will not distinguish between them here. One then enters the main accretion phase during which the central object builds up its mass $\left(M_{\star}\right)$ from a surrounding infalling envelope (of mass $M_{e n v}$ ) and accretion disk, while progressively warming up. In this paper, we will refer to the composite system consisting of the central object, plus envelope and disk as an accreting protostar. The youngest accreting protostars have $\mathrm{M}_{\mathrm{env}}>>\mathrm{M}_{\star}$, and radiate the accretion luminosity $L_{a c c} \approx G M_{\star} \dot{M}_{a c c} / R_{\star}$. In the 'standard' theory of isolated star formation (see Shu et al. 1987, 1993 for reviews), the collapse initial conditions are taken to be (static) singular isothermal spheres or spheroids ( $\rho \sim\left(a^{2} / 2 \pi G\right) r^{-2}-\mathrm{cf}$. Li \& Shu 1996, 1997), there is no runaway collapse phase, and the accretion rate $\dot{M}_{a c c}$ is constant in time $\sim a^{3} / G$, where $a$ is the effective isothermal sound speed. With other collapse initial conditions, the accretion rate is generally timedependent (see $\S 4$ below).

Observations have shown that the main accretion phase is always accompanied by a powerful ejection of a small fraction of the accreted material in the form of prominent bipolar jets/outflows (e.g. Bachiller 1996; Cabrit, this volume). These outflows are believed to carry away the excess angular momentum of the infalling matter (e.g. Königl \& Pudritz 2000, Ferreira, this volume). When the central object has accumulated most ( $\gtrsim 90 \%$ ) of its final, main-sequence mass, it becomes a pre-main sequence (PMS) star, which evolves approximately at fixed mass on the Kelvin-Helmholtz contraction timescale (e.g. Stahler \& Walter 1993). Note that, during the protostellar accretion phase, stars more massive than a few $0.1 M_{\odot}$ start burning deuterium, while stars with masses in excess of $\sim 8 M_{\odot}$ begin to burn hydrogen (see Palla \& Stahler 1991). (There is thus no PMS phase for stars more massive than $\sim 8 M_{\odot}$.)

\subsection{Empirical evolutionary sequence}

In parallel to these theoretical phases, observations have identified several empirical evolutionary stages in the path leading from molecular cloud to (low-mass) main sequence star (e.g. Lada 1987, André, Ward-Thompson, \& Barsony 2000 - AWB2000 -, see also Fig. 1). Historically, these observational stages have been established backward in time, starting from the most evolved, optically visible young stars, and going progressively toward less evolved, heavily obscured objects.

\subsubsection{Infrared YSO classes}

In the near-/mid-infrared, three broad classes of young stellar objects (YSOs) can be distinguished based on the slope $\alpha_{\mathrm{IR}}=\mathrm{d} \log \left(\lambda \mathrm{F}_{\lambda}\right) / \mathrm{d} \log (\lambda)$ of their SEDs between $2.2 \mu \mathrm{m}$ 


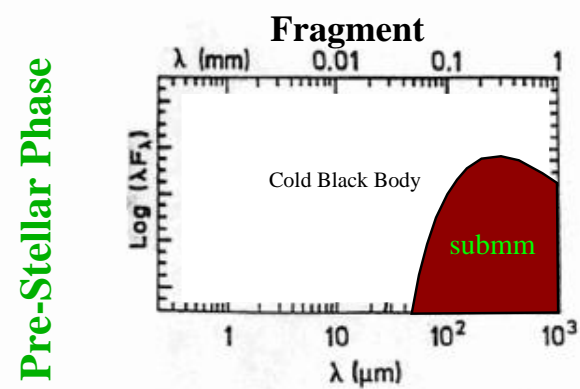

Formation of the central protostellar object
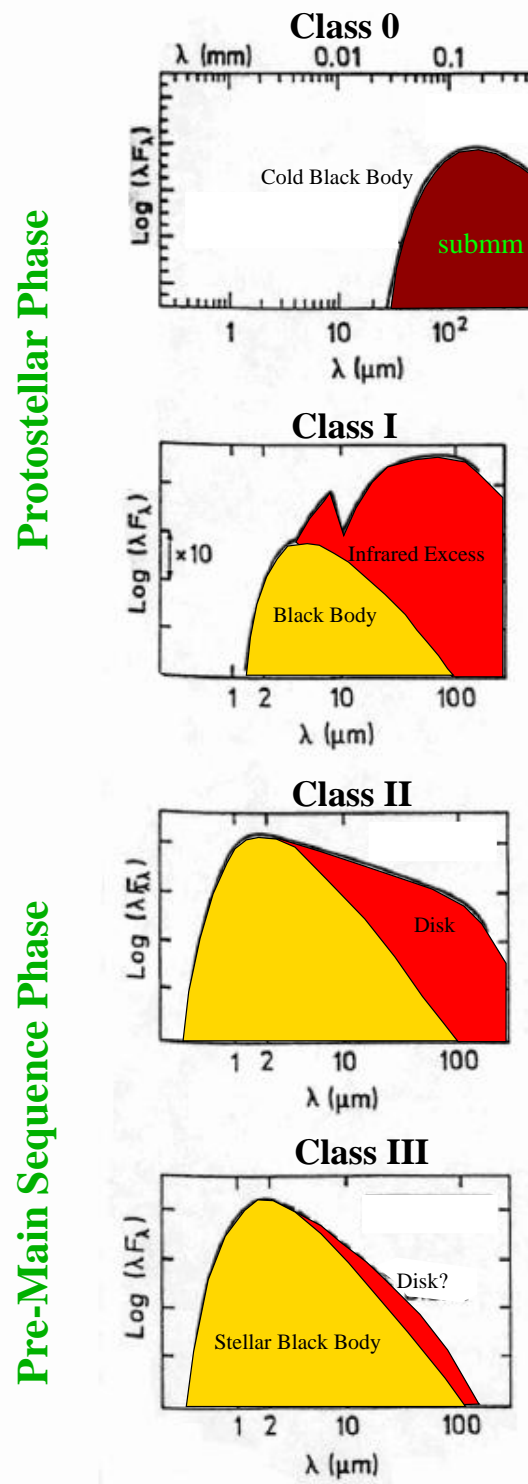

Parent Cloud
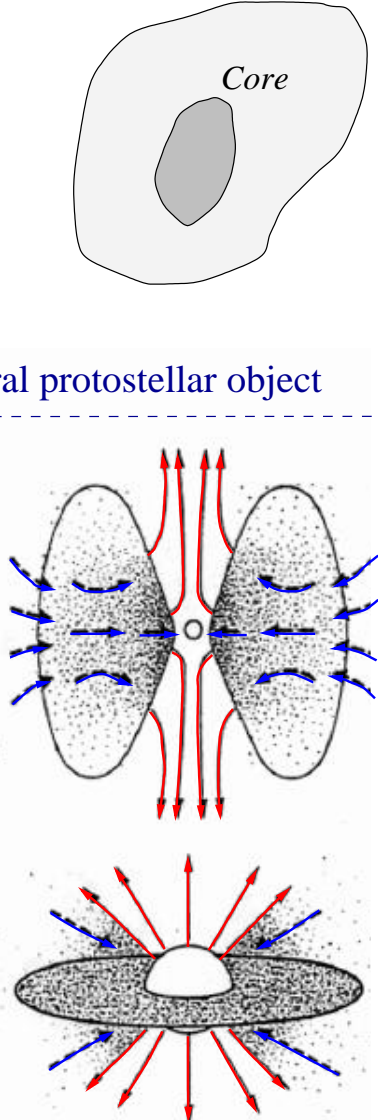

Birthline for

Pre-main sequence stars

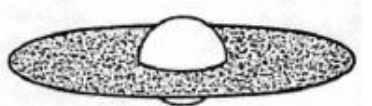

Protoplanetary Disk ?

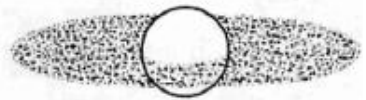

Debris + Planets ? $\mathrm{t} \sim 0 \mathrm{yr}$

Young Accreting Protostar

$\mathrm{T}_{\text {bol }}<70 \mathrm{~K}, \mathrm{M}_{*} \ll \mathrm{M}_{\mathrm{env}}$

$<30000 \mathrm{yr}$

Evolved Accreting Protostar

Pre-Stellar Dense Core

$\mathrm{T}_{\mathrm{bol}} \sim 10-20 \mathrm{~K}, \mathrm{M}_{*}=0$

1000000 yr

$\mathrm{T}_{\text {boil }}$ 70-650 K, $\mathrm{M}_{*}>\mathrm{M}_{\mathrm{env}}$

$\sim 200000 \mathrm{yr}$

Classical T Tauri Star

$\mathrm{T}_{\text {bol }} \sim 650-2880 \mathrm{~K}, \mathrm{M}_{\text {Disk }} \sim 0.01 \mathrm{M}_{\odot}$

$\sim 1000000 \mathrm{yr}$

Weak T Tauri Star

$\mathrm{T}_{\text {bol }}>2880 \mathrm{~K}, \mathrm{M}_{\text {Disk }}<\mathrm{M}_{\text {Jupiter }}$

$\sim 10000000 \mathrm{yr}$

Time

Figure 1: Empirical evolutionary sequence for the formation of a single star from a prestellar cloud core to a Class III YSO, based on the shape of the SED (left), the bolometric temperature and the mass of circumstellar material indicated on the right (from Lada 1987; André, WardThompson, \& Barsony 1993; and Chen, Myers et al. 1995). 
and 10-25 $\mu \mathrm{m}$ (Lada \& Wilking 1984, Lada 1987). Going backward in time, Class III $\left(\alpha_{\mathrm{IR}}<-1.5\right)$ and Class II $\left(-1.5<\alpha_{\mathrm{IR}}<0\right)$ sources correspond to PMS stars ("Weak" and "Classical" T Tauri stars, respectively) surrounded by a circumstellar disk (optically thin and optically thick at $\lambda \lesssim 10 \mu \mathrm{m}$, respectively), but lacking a dense circumstellar envelope (see André \& Montmerle 1994 - hereafter AM94). The youngest YSOs detected at $2 \mu \mathrm{m}$ are the Class I sources, which are characterized by $\alpha_{\mathrm{IR}}>0$ (e.g. Wilking, Lada, \& Young 1989), and the close association with dense molecular gas (e.g. Myers et al. 1987). Class I objects are interpreted as relatively evolved protostars with typical ages $\sim 1-2 \times 10^{5}$ yr (e.g. Greene et al. 1994, Kenyon \& Hartmann 1995), surrounded by both a disk and a low-mass (often only residual) circumstellar envelope (AM94, Lucas \& Roche 1997, Terebey et al. 2001). To first order, their SEDs are successfully modeled in the framework of the 'standard' theory of isolated protostars (e.g. Adams, Lada, \& Shu 1987, Kenyon et al. 1993), in agreement with the idea that they derive a substantial fraction of their luminosity from accretion (e.g. Greene \& Lada 1996 and Kenyon et al. 1998).

\subsubsection{Class 0 protostars}

Besides these infrared YSOs, (sub)millimeter dust continuum mapping of molecular cloud cores with large ground-based radiotelescopes has revealed a wealth of cold, compact protostellar condensations which often remain totally invisible at $\lambda \lesssim 25 \mu \mathrm{m}$ (see, e.g., AWB2000 for a review). Some of these condensations are associated with formed (i.e., hydrostatic) YSOs and have been designated "Class 0" protostars (André, Ward-Thompson, \& Barsony 1993 - AWB93). Specifically, Class 0 objects are defined by the following observational properties (AWB93):

- (1) Indirect evidence for a central YSO, as indicated by, e.g., the detection of compact centimeter radio continuum emission or the presence of a collimated $\mathrm{CO}$ outflow.

- (2) Centrally peaked but extended submillimeter continuum emission tracing the presence of a spheroidal circumstellar dust envelope (as opposed to just a disk).

- (3) High ratio of submillimeter to bolometric luminosity: $\mathrm{L}_{s u b m m} / \mathrm{L}_{b o l}>>0.5 \%$, where $\mathrm{L}_{\text {submm }}$ is measured longward of $350 \mu \mathrm{m}$. [In practice, this often means a "cold" spectral energy distribution (SED) with a bolometric temperature $T_{\text {bol }}<70 \mathrm{~K}-\mathrm{cf}$. Chen et al. 1995.]

Property (1) distinguishes Class 0 objects from prestellar sources (see $\S 1.2 .3$ below), since deep radio continuum VLA observations reveal no compact radio continuum emission at the center of starless cores (Bontemps 1996, Yun et al. 1996).

Properties (2) and (3) distinguish Class 0 objects from more evolved (e.g. Class I and Class II) YSOs detected in the near- to mid-infrared (cf. $\S 1.2 .1$ ). In particular, the ratio $\mathrm{L}_{s u b m m} / \mathrm{L}_{b o l}$ of submm to bolometric luminosity should roughly track the ratio $M_{e n v} / M_{\star}$ of envelope to stellar mass, and may be used as a quantitative evolutionary indicator (decreasing with time) for self-embedded YSOs. The Class 0 condition $\mathrm{L}_{s u b m m} / \mathrm{L}_{b o l}>0.5 \%$ approximately selects objects which have a ratio of envelope to stellar mass $M_{e n v} / M_{\star}>1$ (AWB93, AM94). [A roughly equivalent criterion is $M_{\text {env }} / L_{b o l}>0.1 M_{\odot} / L_{\odot} \cdot$ ] Class 0 objects are thus excellent candidates for being very young accreting protostars in which a hydrostatic core has already formed but not yet accreted the majority of its final mass.

About 30-40 confirmed Class 0 objects have been identified up to now (see Table I of AWB2000). These objects appear to have strong and more frequent infall signatures than Class I sources (Gregersen et al. 1997, Mardones et al. 1997, see also $\S 3.2$ below), confirming their interpretation as very young protostars. The lifetime of the Class 0 phase is apparently short ( $\lesssim 3 \times 10^{4}$ yr - e.g., AM94), compared to the Class I near-IR phase $\left(\sim 2 \times 10^{5} \mathrm{yr}-\right.$ see $\S 1.2 .1$ above). However, this lifetime is presently uncertain and may well vary from cloud to cloud (the quoted value corresponds to a rough average estimate). More complete submillimeter surveys would be required to get at better estimates. 

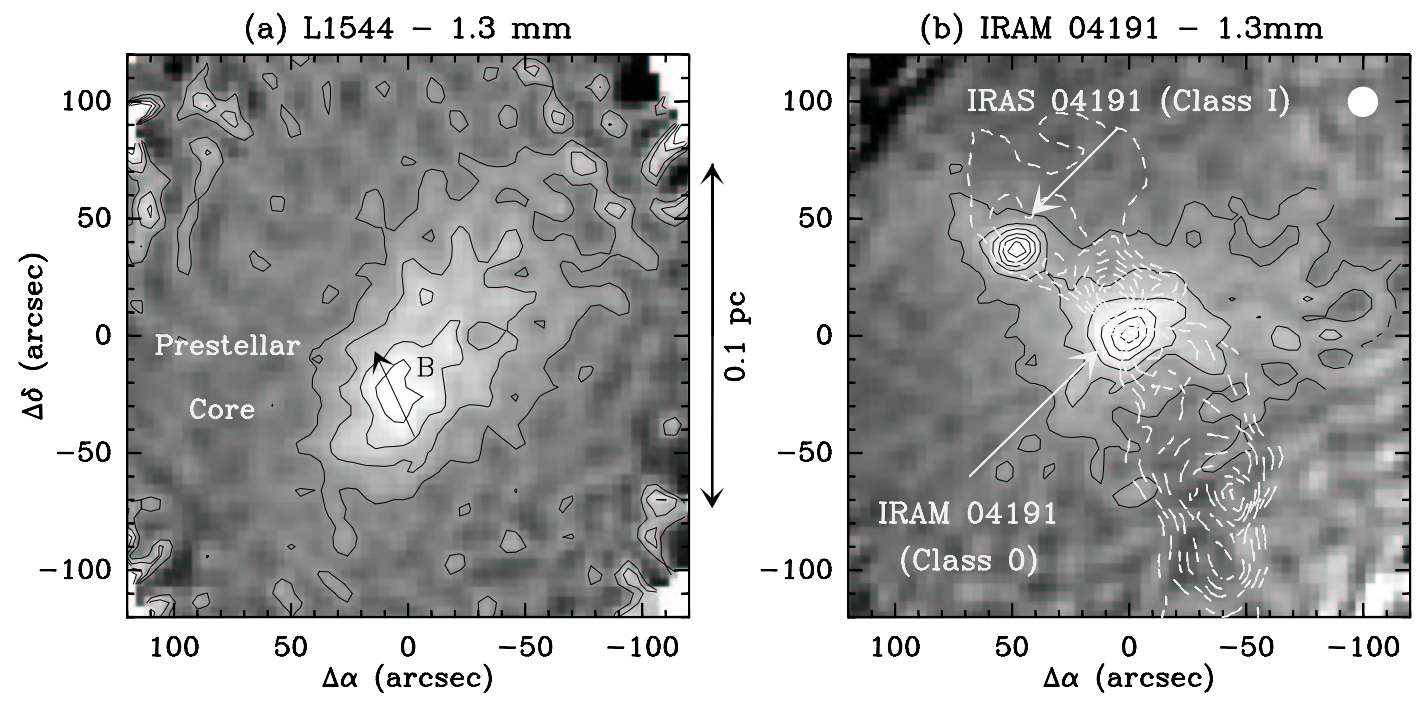

Figure 2: Dust continuum maps of L1544 (a) and IRAM 04191 (b) at $1.3 \mathrm{~mm}$ taken with the IRAM $30 \mathrm{~m}$ telescope and the MPIfR bolometer array in the on-the-fly dual-beam scanning mode (from Ward-Thompson et al. 1999 and André et al. 1999, respectively). Effective angular resolution: 13"; base contour and contour step: $20 \mathrm{mJy} /$ beam. The direction of the magnetic field measured in L1544 with the SCUBA polarimeter on JCMT (Ward-Thompson et al. 2000) and the collimated $\mathrm{CO}(2-1)$ outflow emanating from IRAM 04191 are shown.

Although it has been pointed out that some Class I sources may look like Class 0 objects when observed nearly edge-on (e.g. Yorke, Bodenheimer, \& Laughlin 1995; Masunaga \& Inutsuka 2000), it is important to stress that the two types of objects can be distinguished unambiguously when maps of optically thin (sub)mm continuum emission are available in addition to SEDs. Such maps provide direct evidence for massive, spatially resolved circumstellar envelopes around bona fide Class 0 objects (see, e.g., Fig. 2b), while less massive (often only residual) envelopes are seen in the case of Class I sources regardless of orientation (e.g. AM94, Motte \& André 2001).

\subsubsection{Prestellar cores}

(Sub)millimeter line and continuum observations have also detected a large number of starless cloud cores which appear to be prestellar in nature. For convenience, the prestellar stage of star formation may be defined as the phase in which a gravitationally bound core has formed in a molecular cloud, and evolves toward higher degrees of central condensation, but no central hydrostatic protostellar object exists yet within the core (i.e., $\left.M_{\star}=0\right)$.

A pioneering survey of isolated dense cores in dark clouds was carried out in transitions of $\mathrm{NH}_{3}$ by Myers and co-workers (see Benson \& Myers 1989 and references therein), who catalogued about 90 cores. These were separated into starless cores and cores with stars (Beichman et al. 1986), on the basis of the presence or absence of an embedded source detected by IRAS. The starless $\mathrm{NH}_{3}$ cores were identified by Beichman et al. as the potential sites of future isolated low-mass star formation. Other dense core surveys have been carried out by Clemens \& Barvainis (1988), Lee \& Myers (1999), and Jessop \& WardThompson (2000). A recent compilation of $\mathrm{NH}_{3}$ cores was published by Jijina, Myers, \& Adams (1999).

Using the $15 \mathrm{~m}$ James Clerk Maxwell Telescope (JCMT), Ward-Thompson et al. (1994) observed the $800-\mu \mathrm{m}$ dust continuum emission from about 20 starless $\mathrm{NH}_{3}$ cores from the Benson \& Myers list, mapping 4 of the cores, and showed that they have larger 
FWHM sizes than, but comparable masses to the envelopes of Class 0 protostars. This is consistent with starless $\mathrm{NH}_{3}$ cores being the precursors of protostars (see also Mizuno et al. 1994).

Ward-Thompson et al. (1994) also demonstrated that prestellar cores do not have density profiles which can be modeled by a single scale-free power law, but instead have flat inner radial density profiles (see $\S 2.1$ below), suggestive of magnetically-supported cores contracting by ambipolar diffusion (Mouschovias 1991, Ciolek \& Basu 2001, and references therein). Recent molecular line spectroscopy of several prestellar cores (e.g. Tafalla et al. 1998, Myers et al. 2000, Tafalla 2001) appears to support the argument that they are contracting, but more slowly than the infall seen toward Class 0 protostars (see $\S 3.2$ below). Based on the ratio of the number of starless cores to the number stellar cores (cf. Beichman et al. 1986), the lifetime of the prestellar phase is estimated to be $\sim 3 \times 10^{5}-2 \times 10^{6}$ yr depending on central density (Jijina et al. 1999, Jessop \& Ward-Thompson 2000).

\subsubsection{Evolutionary indicators}

The practical diagnostics that one uses to order YSOs and cloud cores along the evolutionary sequence sketched in Fig. 1 are the shape of the SED (e.g. Lada 1987), the average (or bolometric) temperature (e.g. Myers et al. 1998), and the mass of circumstellar material (e.g. AM94, Saraceno et al. 1996). Independently of the details of any protostellar theory, and in a statistical sense at least, one expects young stellar objects to become warmer and to be surrounded by progressively smaller amounts of circumstellar material as they evolve. Accordingly, the peak of the SED moves from the submillimeter for prestellar cores and (cold, deeply embedded) Class 0 protostars, to the far-IR for Class I protostars, to the near-IR and the optical for PMS stars (Class II and Class III sources) (see Fig. 1 and Masunaga \& Inutsuka 2000). In the case of embedded protostars, the decrease of circumstellar mass with time results from the progressive dissipation of the protostellar envelope through accretion and outflow. For instance, in all self-similar isothermal models of collapse (Whitworth \& Summers 1985), including the standard Shu (1977) model, the mass enclosed within a given radius $R$ of the infalling envelope scales approximately as $M_{e n v}(r<R) \propto t^{-1 / 2}$ with time.

The advent of sensitive bolometer arrays such as MAMBO on the IRAM $30 \mathrm{~m}$ telescope and SCUBA on JCMT provides a very effective method of measuring the mass of prestellar cores and YSO circumstellar structures (envelopes and/or disks) by mapping the associated dust continuum emission in the (sub)millimeter range. Thanks to the small dust optical depth at these wavelengths, the measured emission is directly proportional to the dust mass. The total (gas + dust) circumstellar mass may be derived if the gas-to-dust ratio is assumed or calibrated. For an isothermal dust source, the total mass $M(r<R)$ contained within a radius $R$ from the center is related to the flux density $S_{\nu}(\theta)$ measured at (sub)millimeter frequency $\nu$ over a circle of projected angular radius $\theta=R / d$ by:

$$
M(r<R) \equiv \pi R^{2}\langle\Sigma\rangle_{R}=\left[S_{\nu}^{\mathrm{int}}(\theta) d^{2}\right] /\left[\kappa_{\nu} B_{\nu}\left(T_{\text {dust }}\right)\right],
$$

where $\langle\Sigma\rangle_{R}$ is the average mass column density and $d$ is the distance to the source. The dust opacity per unit (gas + dust) mass column density, $\kappa_{\nu}$, is somewhat uncertain, but the uncertainties are much reduced when appropriate dust models are used (see Henning et al. 1995 for a review). For prestellar cores of intermediate densities $\left(n_{H_{2}} \lesssim 10^{5} \mathrm{~cm}^{-3}\right)$, $\kappa_{\nu}$ is believed to be close to $\kappa_{1.3 \mathrm{~mm}}=0.005 \mathrm{~cm}^{2} \mathrm{~g}^{-1}$ at $\lambda=1.3 \mathrm{~mm}$ (e.g. Hildebrand 1983 , Preibisch et al. 1993). In denser cloud cores and protostellar envelopes, grain coagulation and the formation of ice mantles make $\kappa_{\nu}$ a factor of $\sim 2$ larger, i.e., $\kappa_{1.3 \mathrm{~mm}}=0.01 \mathrm{~cm}^{2} \mathrm{~g}^{-1}$ assuming a gas-to-dust mass ratio of 100 (e.g. Ossenkopf \& Henning 1994). A still higher 
value, $\kappa_{1.3 \mathrm{~mm}}=0.02 \mathrm{~cm}^{2} \mathrm{~g}^{-1}$, is recommended in protoplanetary disks (Beckwith et al. 1990, Pollack et al. 1994).

In contrast to the SEDs of Class I and Class II YSOs which are much broader than a single blackbody, indicative of a broad range of emitting dust temperatures (cf. Adams et al. 1987, Bertout, Basri, \& Bouvier 1988), the SEDs of prestellar cores and Class 0 protostars are reasonably well fitted by a single grey-body curve of the form:

$$
S_{\nu}=B_{\nu}\left(T_{\text {dust }}\right)\left[1-\exp \left(-\tau_{\nu}\right)\right] \Delta \Omega,
$$

where $B_{\nu}\left(T_{\text {dust }}\right)$ is the Planck function for a dust temperature $T_{\text {dust }}, \tau_{\nu}=\kappa_{\nu} \Sigma$ is the dust optical depth, and $\Delta \Omega$ is the source solid angle. In this simple modeling, the dust opacity $\kappa_{\nu}$ is assumed to scale as $\nu^{\beta}$ with $\beta=1.5-2$ as usually appropriate in the submillimeter range (e.g. Hildebrand 1983). As an illustration, Fig. 3 shows the SEDs of the prestellar core L1544 and of the Class 0 object IRAM 04191 (both in the Taurus cloud), based on recent $\mathrm{FIR} / \mathrm{submm} / \mathrm{mm}$ observations with $I S O$, JCMT, and IRAM. As this example shows, starless cores tend to be somwehat colder (e.g. $\left\langle T_{\text {dust }}\right\rangle \sim 9 \mathrm{~K}$ for L1544) than the envelopes of Class 0 protostars (e.g. $\left\langle T_{\text {dust }}\right\rangle \sim 13 \mathrm{~K}$ for IRAM 04191). The energy budget of prestellar cores is consistent with them being only externally heated by the local interstellar radiation field (Lehtinen et al. 1998; Ward-Thompson, André, \& Kirk 2001).
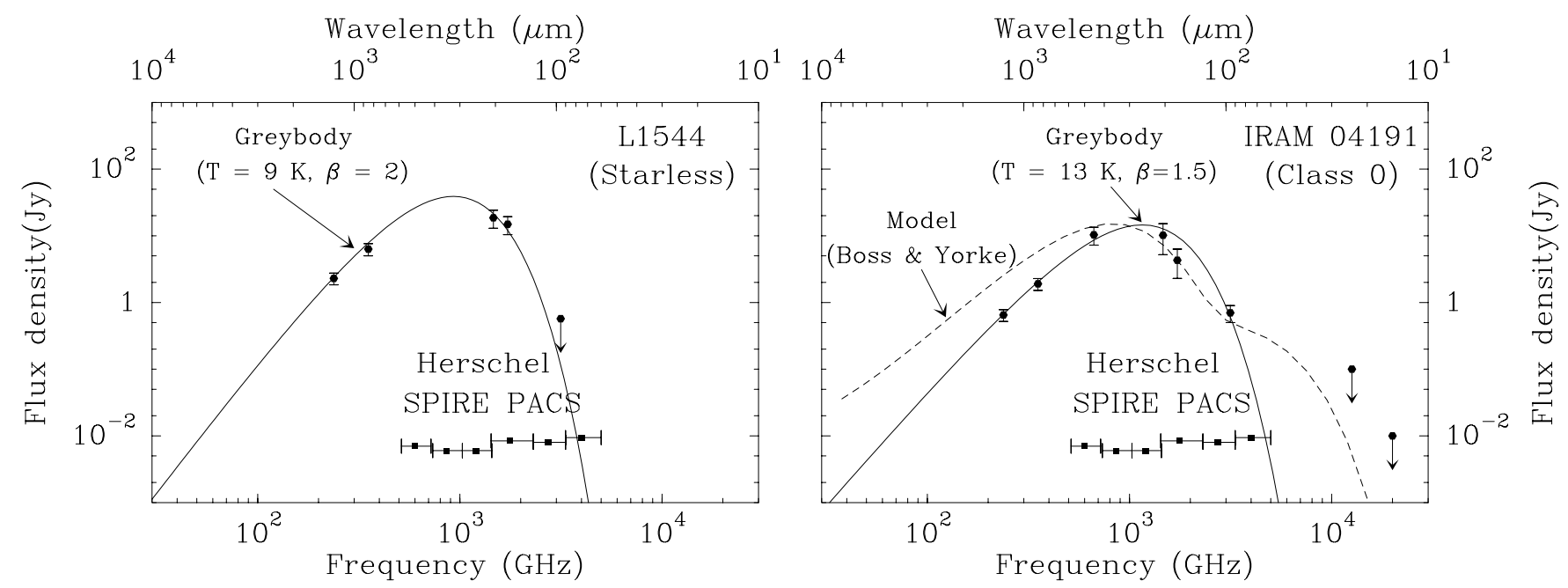

Figure 3: Spectral energy distributions of the prestellar core L1544 (left) and of the Class 0 protostar IRAM 04191 (right), along with grey-body fits (from Ward-Thompson et al. 2001 and André et al. 1999, respectively). The six photometric bands of the SPIRE and PACS imaging instruments on the future FIRST/Herschel space mission are shown, along with their $(10 \sigma$, $1 \mathrm{hr})$ sensitivities. Herschel will be ideally suited to detecting and characterizing all prestellar cores and cold protostars to $\sim 0.03-0.1 M_{\odot}$ and $d \sim 1 \mathrm{kpc}$ in the Galaxy.

Another, potentially very promising indicator is provided by the chemical evolution of cloud cores (e.g. van Dishoeck \& Blake 1998, Langer et al. 2000). However, it will take the next generation of (sub)millimeter instruments (e.g. ALMA) to fully exploit the power of astrochemical differentiation in large samples of objects.

\section{Density structure of individual cloud cores}

Observationally, it is by comparing the (density and velocity) structure of prestellar cores with that of the envelopes surrounding the youngest (Class 0) accreting protostars that 
one may hope to estimate the initial conditions of protostellar collapse.

Two main approaches have been used to trace the density structure of cloud cores: (1) mapping of the optically thin (sub)millimeter continuum emission from the (cold) dust contained in the cores, and (2) mapping of the absorption of the local background infrared emission (from warm cloud dust or remote stars) by the same cold dust component in the cores. Mapping the molecular gas component is generally less effective in this respect as most molecules tend to freeze out onto dust grains in the dense, cold inner parts of cloud cores (e.g. Walmsley et al. 2001).

The first approach makes use of the simple linear relation between the beam-averaged $\mathrm{H}_{2}$ column density, $\left\langle N_{\mathrm{H} 2}\right\rangle_{\text {beam }}$, and the flux density $S_{\nu}^{\text {beam }}$ measured at submillimeter frequencies on any given line of sight:

$$
<N_{\text {H } 2}>_{\text {beam }}=S_{\nu}^{\text {beam }} /\left[\Omega_{\text {beam }} \mu \mathrm{m}_{\mathrm{H}} \kappa_{\nu} B_{\nu}\left(T_{\text {dust }}\right)\right],
$$

where $\Omega_{\text {beam }}$ is the main (Gaussian) beam solid angle, $\mu=2.33$ is the mean molecular weight, and $\mathrm{m}_{\mathrm{H}}$ is the mass of atomic hydrogen. Furthermore, at $\lambda=1.3 \mathrm{~mm}$, the Planck function is often (at least approximately) in the Rayleigh-Jeans regime, and the above equation takes on the practical form:

$$
<N_{\mathrm{H} 2}>\approx 6.5 \times 10^{21} \mathrm{~cm}^{-2} \times\left(\frac{S_{1.3 \mathrm{~mm}}^{\text {beam }}}{10 \mathrm{mJy} / 13^{\prime \prime} \text {-beam }}\right) \times\left(\frac{\mathrm{T}_{\text {dust }}}{15 \mathrm{~K}}\right)^{-1} \times\left(\frac{\kappa_{1.3 \mathrm{~mm}}}{0.005 \mathrm{~cm}^{2} \mathrm{~g}^{-1}}\right)^{-1} .
$$

In the second approach, mostly used with starless cores (e.g. Bacmann et al. 2000, Alves et al. 2001), one seeks to map out the absorption factor, $e^{-\tau_{I R}}$, resulting from dust extinction in the infrared, where $\tau_{I R}$ is the optical depth on a given line of sight:

$$
\tau_{I R}=\kappa_{\mathrm{IR}} \times \Sigma=\kappa_{\mathrm{IR}} \times \mu \mathrm{m}_{\mathrm{H}} \times N_{\mathrm{H} 2} .
$$

\subsection{Prestellar cores}

Although observed prestellar cores are generally not circularly or elliptically symmetric (see, e.g., Fig. 2a), one can still usefully constrain their radial density profiles by averaging the infrared absorption or (sub)millimeter emission they produce in circular or elliptical annuli (see, e.g., Fig. 4).

As an example, Fig. 5a below shows the radial intensity profile $I_{1.3}(\theta)$ derived by circularly averaging the emission in the southeast quadrant of the L1544 map (Fig. 2a). The averaged $1.3 \mathrm{~mm}$ profile similarly obtained for the envelope of the Class 0 protostar L1527 is displayed for comparison, as well as the profile of a singular isothermal sphere (SIS) at $T=10 \mathrm{~K}$ whose density scales as $\rho(r) \propto r^{-2}$. This SIS model profile results from a complete simulation (cf. Appendix of Motte \& André 2001) taking into account both the millimeter continuum observing technique (dual-beam mapping) and the reduction/restoration method (cf. Emerson, Klein, \& Haslam 1979). It can be seen in Fig. 5a that the L1544 profile is significantly flatter than the SIS profile in the inner $\sim 2500$ AU. More generally, assuming spatially uniform dust (temperature and emissivity) properties, the typical prestellar column density profiles derived in this way scale roughly as $N_{H_{2}}(\bar{r}) \propto \bar{r}^{-1}$ with projected radius $\bar{r}$ over the range $\bar{r} \sim 5000-15000 \mathrm{AU}$, and become essentially flat at smaller radii (cf. Ward-Thompson et al. 1994, 1999; André et al. 1996). This is consistent with radial density gradients approaching $\rho(r) \propto r^{-2}$ for $r \sim 5000-15000 \mathrm{AU}$, and flattening to $\rho(r) \propto r^{-1}$ (if the 3-D core shape is spheroidal) or even $\rho(r) \sim$ constant (if the 3-D core shape is disk-like) for $r \leq R_{\text {flat }} \sim 2500-5000$ AU.

More recently, it has been possible to constrain the density gradient of starless cores through absorption studies, both in the mid-IR from space (e.g. Abergel et al. 1996, Bacmann et al. 2000, Siebenmorgen \& Krügel 2000) and in the near-IR from the ground 
(e.g. Alves et al. 2001). In particular, using the ISOCAM infrared camera aboard the ISO satellite, Bacmann et al. (2000) carried out a $7 \mu \mathrm{m}$ survey of 24 low-mass starless dense cores (all undetected by IRAS) and observed absorption features in 23 of them. As an example, Fig. 4 a shows the ISOCAM image (in greyscale) obtained at $6.75 \mu \mathrm{m}$ for the prestellar core L1689B in the Ophiuchus complex. The core is seen in absorption against the diffuse mid-IR background arising from the mean galactic interstellar radiation field (e.g. Mathis et al. 1983) and/or the rear side of the parent molecular cloud (e.g. Bernard et al. 1993). Note the good correspondence between the infrared absorption seen in the ISOCAM image and the $1.3 \mathrm{~mm}$ emission mapped with the IRAM $30 \mathrm{~m}$ telescope (shown as contours in Fig. 4a), confirming that both trace the same cold dust material.
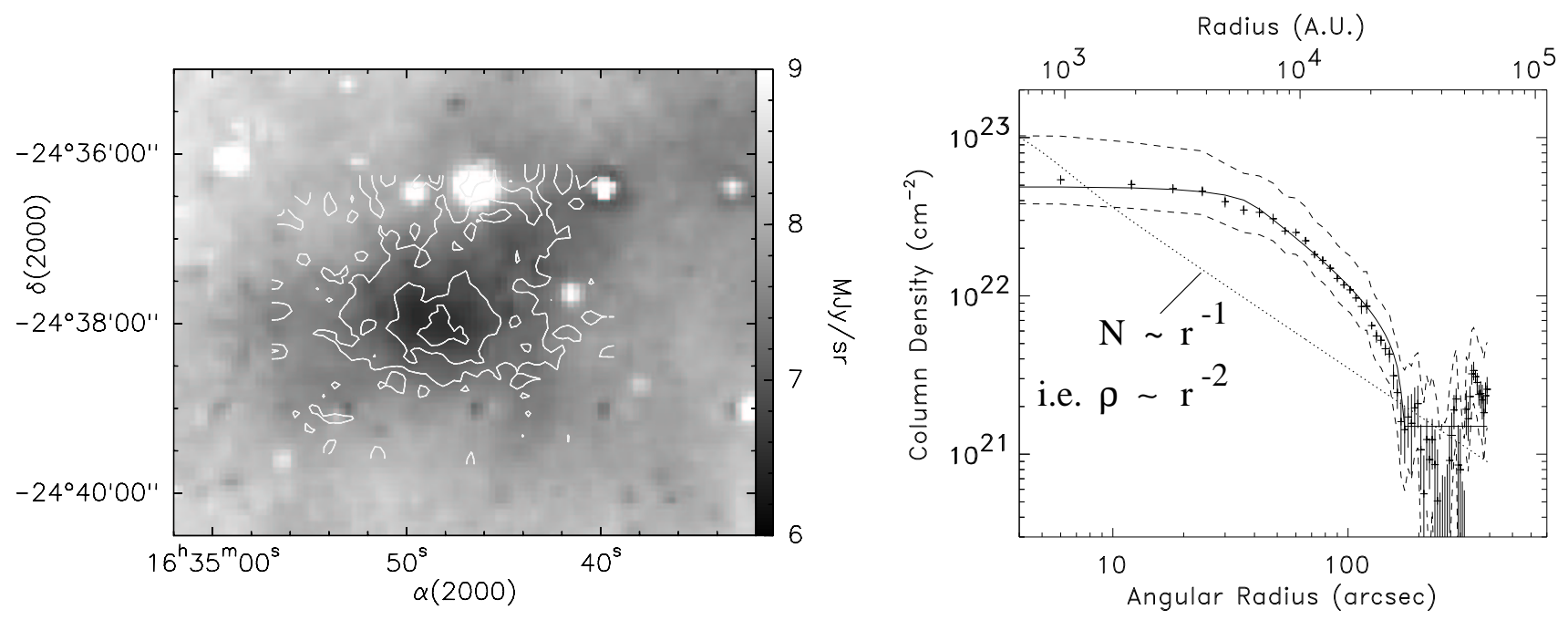

Figure 4: (a) ISOCAM $6.75 \mu \mathrm{m}$ absorption image of the prestellar core L1689B (greyscale indicated on the right in $\mathrm{MJy} / \mathrm{sr}$ ). A $1.3 \mathrm{~mm}$ dust continuum emission map obtained by André et al. (1996) at the IRAM $30 \mathrm{~m}$ telescope is superimposed as contours (levels: 10, 30, $50 \mathrm{mJy} / 13^{\prime \prime}$ beam). (b) Column density profile of L1689B (crosses) derived from the absorption map shown in (a) by averaging the intensity over elliptical annuli for a $40^{\circ}$ sector in the Southern part of the core. The dashed curves limit the range of profiles compatible with the data given the uncertainties affecting the absorption analysis. The dotted line is the $N_{\mathrm{H} 2} \propto \bar{r}^{-1}$ powerlaw profile of a singular isothermal sphere at $T=10 \mathrm{~K}$; the solid curve is a parameterized Bonnor-Ebert-like model (Adapted from Bacmann et al. 2000.)

The column density structure of the absorbing cores can be derived from a simple modeling of the ISOCAM observations. The mid-IR intensity measured at projected radius $\bar{r}$ from core center may be expressed as:

$$
I(\bar{r})=I_{b a c k} \cdot e^{-\tau(\bar{r})}+I_{\text {fore }},
$$

where $I_{b a c k}$ and $I_{\text {fore }}$ are the background and foreground intensities respectively. The foreground includes, and is often dominated by, the zodiacal emission $(\sim 4 \mathrm{MJy} / \mathrm{sr}$ for L1689B). (For simplicity, $I_{b a c k}$ and $I_{\text {fore }}$ are here assumed to be uniform over the region mapped.) The $\mathrm{H}_{2}$ column density, $\mathrm{N}_{H_{2}}$, is given by $N_{H_{2}}=\kappa_{8 \mu} \cdot \tau(\bar{r})$, where $\kappa_{8 \mu}$ is the dust opacity at $\lambda=7.75 \mu \mathrm{m}$ (cf. Draine \& Lee 1984). $\mathrm{I}_{\text {back }}$ and $\mathrm{I}_{\text {fore }}$ are two uncertain quantities, but their sum, $\mathrm{I}_{b a c k}+\mathrm{I}_{\text {fore }}$, is constrained by the mean mid-IR intensity measured in the maps far from the cores. Additional constraints on $\mathrm{I}_{b a c k}$ and $\mathrm{I}_{\text {fore }}$ can be obtained from independent millimeter measurements of the $\mathrm{H}_{2}$ column density in the cores: Millimeter continuum maps provide good estimates of the central column densities, $\mathrm{N}_{c e n}$, accurate to a factor of $\sim 2 ; \mathrm{C}^{18} \mathrm{O}(1-0)$ line observations provide estimates (also accurate to a factor of $\sim 2$ ) of the column density $\mathrm{N}_{\text {out }}$ of the cores at large radii. 
Using these various constraints on $\mathrm{I}_{b a c k}$ and $\mathrm{I}_{\text {fore }}$, a range of possible column density profiles can be derived for each core imaged in absorption with ISOCAM. This is shown in Fig. 4b for L1689B. In Fig. 4b, the crosses display the 'best' estimate of the column density profile of $\mathrm{L} 1689 \mathrm{~B}$, derived using $\mathrm{N}_{c e n} \sim 4 \times 10^{22} \mathrm{~cm}^{-2}$ in the flat inner region and $\mathrm{N}_{\text {out }} \sim 2 \times 10^{21} \mathrm{~cm}^{-2}$ at $\sim 0.16 \mathrm{pc}$, as estimated from millimeter data (cf. André et al. 1996 and Bacmann et al. 2000). The dashed curves lying above and below the 'best' profile limit the range of column density profiles that are consistent with the mid-IR absorption data, given the permitted range of values for $I_{b a c k}$ and $I_{\text {fore }}$.

In general, four different regimes can be distinguished on the prestellar column density profiles (see the case of L1689B in Fig. 4b):

a) a flat inner region, b) a region roughly consistent with $N_{\mathrm{H} 2} \propto \bar{r}^{-1}$ (corresponding to $\rho \propto r^{-2}$ for a spheroidal core), c) an edge where the column density falls off typically more rapidly than $N_{\mathrm{H} 2} \propto \bar{r}^{-2}$ with projected radius (suggesting a density gradient steeper than $\rho \propto r^{-3}$ ), until d) the end of the the core is reached and $N_{\mathrm{H} 2}$ fluctuates about the mean value $N_{\text {out }} \sim 2 \times 10^{21} \mathrm{~cm}^{-2}$ characterizing the ambient molecular cloud.

Thus, the inner flattening of prestellar cores observed in submillimeter emission (see above) is confirmed by the mid-IR absorption observations. Furthermore, a new result emerges: at least in some cases, such as L1689B, isolated prestellar cores appear to be characterized by sharp edges defining typical outer radii $R_{\text {out }} \sim 20000 \mathrm{AU} \sim 0.1 \mathrm{pc}$.

\subsubsection{Comparison with theroretical models}

These observed features, i.e., inner flattening and sharp outer edge, set constraints on possible models of core structure. First of all, it is clear that self-similar, singular models such as the singular isothermal sphere and magnetized, non-spherical generalizations of it (e.g. Shu 1977, Li \& Shu 1996), cannot account for the detailed radial density structure of prestellar cores, since they match neither the flat inner portion nor the sharp edge seen in the observed column density profiles. Morphologically, the profiles observed by Bacmann et al. (2000) can be reasonably well fitted by finite-sized sphere models with $\rho=\rho_{c}$ for $r<R_{\text {in }}$ and $\rho \propto r^{-2}$ for $R_{\text {in }}<r<R_{\text {out }}$, where $R_{\text {in }} \sim 0.01-0.04 \mathrm{pc}$ and $R_{\text {out }} \sim 0.05-0.5 \mathrm{pc}$. Such simple parameterized models mimic the density structure of pressure-bounded, selfgravitating isothermal Bonnor-Ebert spheres (e.g. Bonnor 1956). It should be pointed out, however, that most starless cores exhibit elongated shapes (see, e.g., Fig. 2a) which are not consistent with spherical models beyond $\sim 0.03 \mathrm{pc}$. Furthermore, the inferred density contrasts (from center to edge) are generally larger (i.e., $\gtrsim 20-80$ ) than the maximum contrast of $\sim 14$ for a stable Bonnor-Ebert sphere. This suggests that the cores cannot be described as simple isothermal hydrostatic structures and are either already contracting or experiencing extra support from static or turbulent magnetic fields (e.g. Curry \& McKee 2000).

A natural way of accounting for flat inner density gradients, high density contrasts, and sharp edges is to consider models of cores threaded by a static magnetic field and evolving through ambipolar diffusion (e.g. Basu \& Mouschovias 1995, Ciolek \& Mouschovias 1995). In these models, at any given time prior to protostar formation, the cores are expected to feature a uniform-density central region whose size corresponds to the instantaneous Jeans length. This agrees well with the characteristics of the flat inner regions seen in starless cores. Furthermore, the observed sharp edges (e.g. Fig. 4b) are consistent with the model predictions (shortly) after the formation of a magnetically supercritical core. This is because when a supercritical core forms, it collapses dynamically inward, while the outer (subcritical) envelope is still efficiently supported by the magnetic field and remains essentially "held in place". As a result, a steep density profile develops at the outer boundary of the supercritical core.

A notable problem, however, with these ambipolar diffusion models involving only a static 
magnetic field is that they require fairly large field strengths $(\sim 30-100 \mu \mathrm{G}$, see Bacmann et al. 2000), which seem to exceed existing Zeeman estimates (typically $\lesssim 10 \mu \mathrm{G}$ for low-mass dense cores - e.g. Crutcher 1999, Crutcher \& Troland 2000). It is possible that more elaborate versions of the models, incorporating the effects of a non-static, turbulent magnetic field in the outer parts of the cores, would be more satisfactory and could also account for the filamentary shape often seen on large $(\gtrsim 0.25 \mathrm{pc})$ scales.

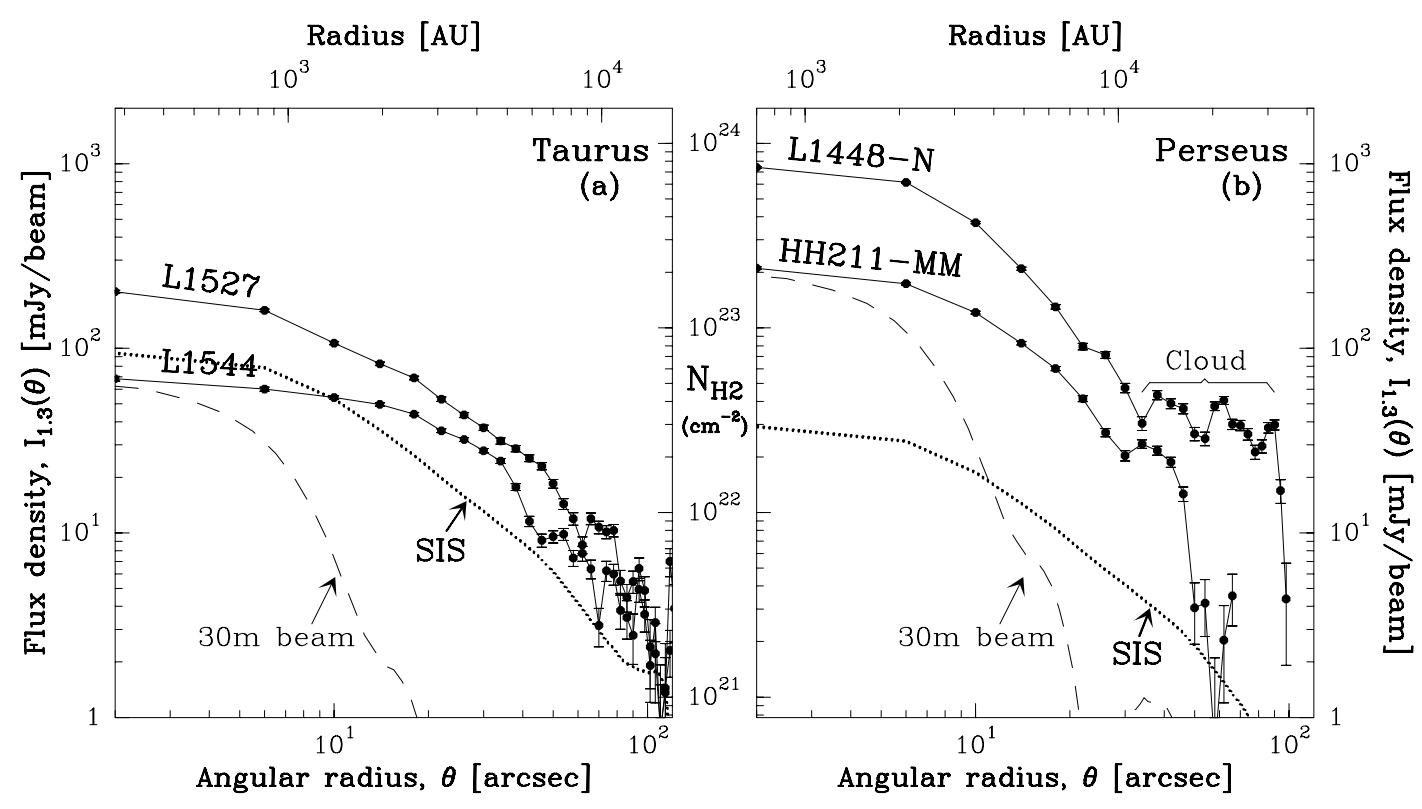

Figure 5: Averaged $1.3 \mathrm{~mm}$ radial intensity profiles of (a) L1544 (prestellar) and L1527 (Class 0) in Taurus $(d=140 \mathrm{pc}$ ), and (b) HH211-MM and L1448-N (Class 0s) in Perseus $(d=350 \mathrm{pc}$ ), compared to the simulated profile of a singular isothermal sphere (SIS) model at $T=10 \mathrm{~K}$. The profile of the $30 \mathrm{~m}$ beam is shown. An approximate column density scale, calculated assuming representative dust properties $\left(T_{d}=15 \mathrm{~K}, \kappa_{1.3}=0.0075 \mathrm{~cm}^{2} \mathrm{~g}^{-1}\right)$ is also indicated. (Adapted from Ward-Thompson et al. 1999 and Motte \& André 2001.)

\subsection{Protostellar envelopes}

In contrast to prestellar cores, protostellar envelopes are always found to be strongly centrally condensed (see, e.g. Fig. 2b) and do not exhibit any marked inner flattening in their radial (column) density profiles. In practice, this means that, when protostars are mapped with the resolution of the largest single-dish telescopes, the measured peak flux density is typically a fraction $\gtrsim 20 \%$ of the flux integrated over five beam widths. This is illustrated in Fig. 5 which shows the circularly-averaged radial intensity profiles measured at $1.3 \mathrm{~mm}$ for the circumstellar envelopes of the Class 0 objects L1527 in Taurus and HH211-MM and L1448-N in Perseus (Motte \& André 2001). (Note that the inner disk contribution has been subtracted from the profiles based on high-resolution interferometric measurements.) Apart from a small overdensity factor $(\sim 2)$, the profile of the L1527 envelope closely follows the shape of the SIS $\rho(r) \propto r^{-2}$ simulated profile (see Fig. 5a), and is significantly steeper than the L1544 prestellar profile at small radii.

More generally, several (sub)millimeter continuum mapping studies indicate that protostellar envelopes in regions of isolated star formation such as Taurus have radial density gradients generally consistent with $\rho(r) \propto r^{-p}$ with $p \sim 1.5-2$ over more than $\sim 10000$ $15000 \mathrm{AU}$ in radius (e.g. Ladd et al. 1991, Chandler \& Richer 2000, Hogerheijde \& 
Sandell 2000, Shirley et al. 2000, Motte \& André 2001). In particular, Motte \& André (2001) mapped the complete sample of Taurus Class I infrared sources (Kenyon \& Hartmann 1995) at $1.3 \mathrm{~mm}$ with the MPIfR bolometer arrays on the IRAM $30 \mathrm{~m}$ telescope (see, e.g., Fig. $2 \mathrm{~b}$ and Fig. 5). Notice that, to first order, the envelopes of low-luminosity protostars $\left(L_{b o l} \lesssim 1-10 L_{\odot}\right)$, including most Taurus embedded YSOs, should be roughly isothermal (at the temperature $\mathrm{T} \simeq 10 \mathrm{~K}$ of the parent cloud) over the range of radii $(r \sim 1000-15000 \mathrm{AU})$ probed by IRAM 30 m maps.

The density gradient estimated for bona-fide (Class 0 and Class I) protostars (i.e., $p \sim 1.5-2$ ) agrees with most collapse models which predict a value of $p$ between 2 and 1.5 during the protostellar accretion phase (before and after the passage of the collapse expansion wave, respectively - see $\S 4$ below). Furthermore, the median envelope mass, $\overline{M_{e n v}}(r<4200 A U) \sim 0.3 M_{\odot}$, measured for the bona-fide protostars of Taurus is consistent with the predictions of the 'standard' inside-out collapse model (e.g. Shu et al. 1987) for $\sim 10^{5}$ yr-old protostars.

The situation is markedly different in regions where stars form in tight groups or clusters, such as Serpens, Perseus, and the $\rho$ Ophiuchi main cloud. In this case, the observed protostellar envelopes are clearly not scale-free: they merge with dense cores, other envelopes, and/or the diffuse ambient cloud at a finite radius $R_{\text {out }} \lesssim 5000 \mathrm{AU}$ (Motte, André, \& Neri 1998 - MAN98; Motte \& André 2001). Moreover, as can be seen in Fig. 5b for HH211-MM and L1448-N, the Class 0 envelopes mapped in regions of multiple star formation are found to be 3 to 12 times denser than a SIS at $\mathrm{T}=10 \mathrm{~K}$ (which is the typical gas kinetic temperature expected in these clouds prior to massive star formation - see Goldsmith \& Langer 1978 and Evans 1999). Static magnetic fields can only account for moderate overdensity factors $(\lesssim 2$ ) compared to an unmagnetized SIS (see Li \& Shu 1997). Turbulence could in principle contribute to the support of the initial dense core (e.g. Myers \& Fuller 1992, Mardones et al. 1997). However, the small-scale condensations corresponding to the precursors of protostars in star-forming clusters (e.g. MAN98) appear to be largely devoid of turbulence (Belloche et al. 2001a - see $\S 3.1$ below). A more likely explanation in this case is that the collapse started from non-singular initial conditions, resulting in a non-equilibrium density configuration with a large overdensity factor $(\lesssim 10$ ) and significant inward velocities near $t \sim 0$ (as in the Larson-Penston similarity collapse flow, see $\S 4$ below). Follow-up line observations of the envelopes' velocity fields are nevertheless required to confirm this suggestion.

\section{Velocity structure}

Probing the velocity field of prestellar cores and protostellar envelopes is more difficult and more time-consuming than measuring their density structure, especially since the full kinematic pattern consists of several distinct velocity components: e.g., turbulence, inflow, rotation, and (in protostars) outflow. In the case of protostars, the outflow component is often overwhelming, being associated with the highest velocities ( $\gtrsim 10 \mathrm{~km} \mathrm{~s}^{-1}$ ) over the largest spatial extents (up to $\gtrsim 1 \mathrm{pc}$ ) (see, e.g., Cabrit, this volume). This obviously complicates the quantitative modeling of the other components. Furthermore, only motions along the line of sight produce Doppler shifts, and the observed line profiles correspond to averages over the telescope beam and over the line of sight.

\subsection{Turbulence}

On large $(\gtrsim$ pc) scales, the spectral line widths observed in molecular clouds indicate highly supersonic motions (e.g. Barrett, Meeks, \& Weinreb 1964; Falgarone \& Pety 2001). The supersonic or nonthermal component of the line width, $\sigma_{N T}$, roughly follows 
the scaling law $\sigma_{N T} \propto R^{0.5}$ (e.g. Larson 1981, Myers 1983, Fuller \& Myers 1992), and is believed to arise from MHD turbulence, i.e., hydromagnetic waves (e.g. Arons \& Max 1975, Mouschovias \& Psaltis 1995). The dissipation of a significant fraction of this nonthermal turbulent support on small $(<0.1 \mathrm{pc})$ scales is a prerequesite for the formation of prestellar cores (e.g. Nakano 1998). Observations indeed suggest that prestellar cores are "coherent" structures within which the turbulent contribution to the line width is small (e.g. subsonic) and nearly independent of radius (Goodman et al. 1998 - see Fig. 6).
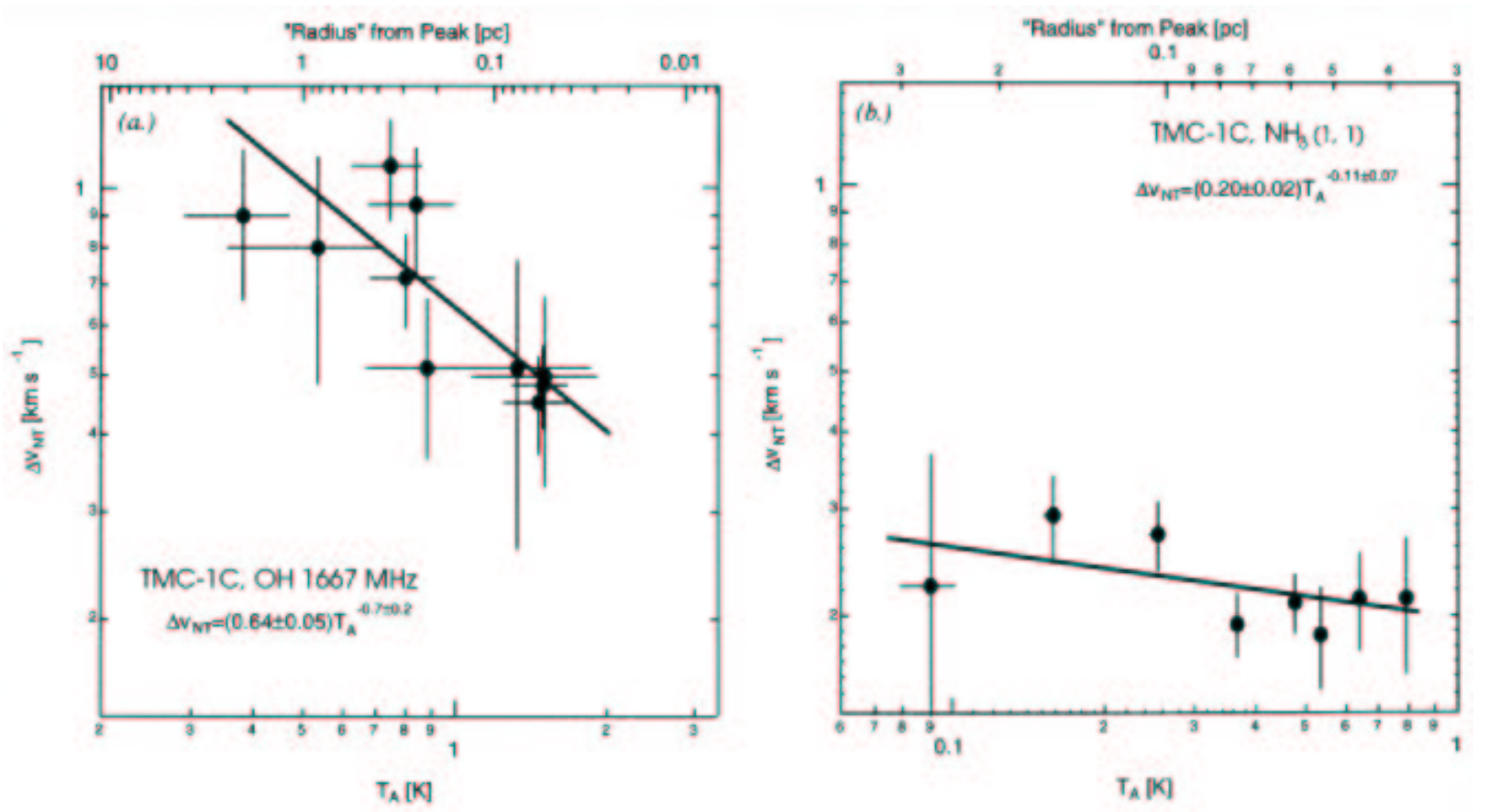

Figure 6: Nonthermal line width as a function of radius (upper scale, increasing to the left) and antenna temperature (lower scale) in the Taurus starless core TMC-1C derived from $\mathrm{OH}$ (a) and $\mathrm{NH}_{3}$ (b) observations. Note that the nonthermal component of the line width is nearly independent of radius in the dense inner $\sim 0.1 \mathrm{pc}$ region traced by $\mathrm{NH}_{3}$ (right panel), while a power-law dependence $\left(\Delta V_{N T} \propto R^{0.3}\right)$ is observed in the outer $\sim 1$ pc region traced by $\mathrm{OH}$. (From Goodman et al. 1998).

Part of the turbulence in molecular clouds originates from the formed protostars themselves which re-inject copious amounts of kinetic energy into the ISM through their powerful outflows (e.g. Norman \& Silk 1980, Matzner \& McKee 2000). Accordingly, at a given size, dense cores with embedded (Class 0 or Class I) protostars tend to have broader line widths than starless cores (e.g. Fuller \& Myers 1992, Zhou et al. 1994).

On intermediate $(\sim 0.1 \mathrm{pc})$ scales, there are strong differences between the "low-mass" cores observed in regions of distributed star formation (e.g. Taurus), whose line widths are dominated by thermal motions (e.g. Benson \& Myers 1989), and the "massive", cluster-forming cores of, e.g., Ophiuchus and Orion, which have line widths dominated by turbulent motions (e.g. Caselli \& Myers 1995). It should be pointed out, however, that the small $(\sim 0.03 \mathrm{pc})$ prestellar condensations of the Ophiuchus, Serpens, Perseus, and Orion cluster-forming regions are characterized by very narrow line widths (e.g. Belloche et al. 2001a, Myers 2001) reminiscent of the thermal cores of Taurus. In the $\rho$ Oph protocluster, for instance, the nonthermal velocity dispersion is about half the thermal velocity dispersion of $\mathrm{H}_{2}\left(\sigma_{N T} / \sigma_{T} \sim 0.7\right)$ toward the starless condensations of Oph B1, C, E, F (Belloche et al. 2001a). This suggests that, even in cluster-forming clouds, the initial conditions of individual protostellar collapse are coherent and essentially free of turbulence. The most direct effect of the level of large-scale turbulence in a cloud may be 
to define the fragmentation lengthscale over which local collapse is possible (e.g. Myers 1998, Klessen et al. 2000).
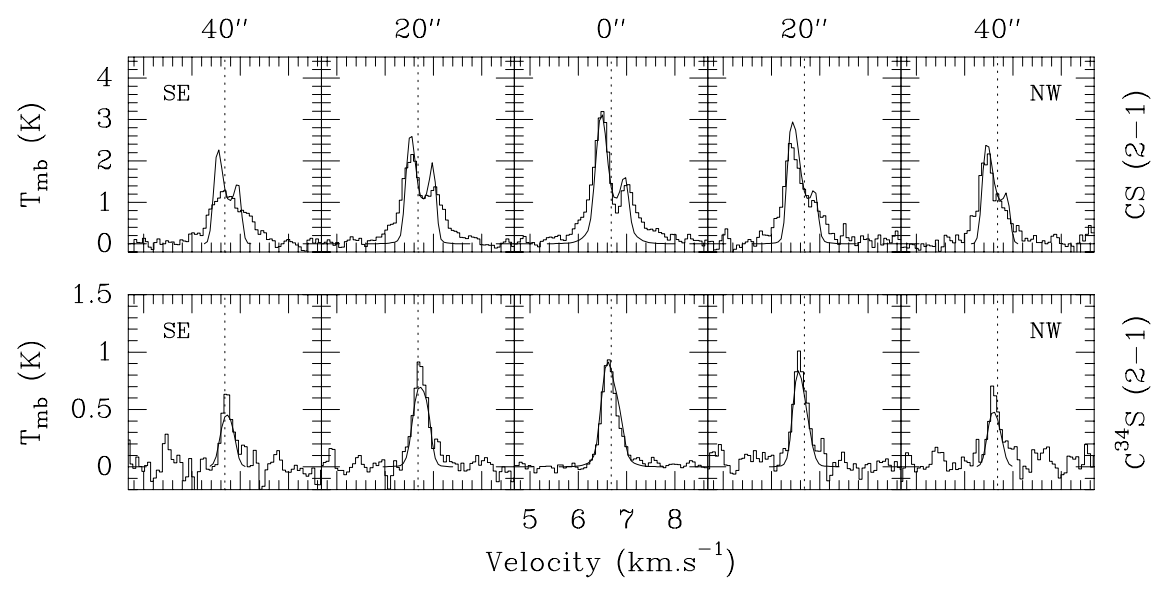

Figure 7: Spectra observed with the IRAM 30m telescope toward the Taurus Class 0 protostar IRAM 04191, along the axis perpendicular to the outflow, in the optically thick CS $(2-1)$ and optically thin $\mathrm{C}^{34} \mathrm{~S}(2-1)$ lines. Synthetic spectra corresponding to a 'spherical' collapse model including rotation with $V_{\text {inf }}(700 \mathrm{AU})=0.4 \mathrm{~km} \mathrm{~s}^{-1}, V_{\text {inf }}(2000 \mathrm{AU})=0.1 \mathrm{~km} \mathrm{~s}^{-1}$, and $V_{\text {rot }}(2000 \mathrm{AU})=0.2 \mathrm{~km} \mathrm{~s}^{-1}$ are shown. (From Belloche et al. 2001b.)

\subsection{Infall}

The envelopes of Class 0 protostars were the first low-mass dense cores for which convincing spectroscopic signatures of gravitational infall motions could be obtained, confirming their protostellar nature (e.g. Walker et al. 1986, Zhou et al. 1993, Gregersen et al. 1997, Mardones et al. 1997, Gregersen et al. 2000).

Inward motions can be traced by optically thick molecular lines which should (locally) exhibit asymmetric self-absorbed profiles skewed to the blue (e.g. Evans 1999; Myers, Evans, \& Ohashi 2000 - see example in Fig. 7). The origin of this characteristic "infall asymmetry" is, qualitatively at least, easy to understand. At any given velocity $v$, the strength of an optically thick spectral line approximately reflects the excitation temperature, $T_{e x}$, at the position where $\tau(v) \sim 1$ along the line of sight. As star-forming cores are generally centrally-condensed, molecular lines tend to be more excited closer to core center: there is a gradient in excitation temperature, $T_{e x}$, toward the center. In the case of inward motions, it is easy to see that the $\tau(v)=1$ point on the line of sight is located farther away from core center, and thus has a lower $T_{e x}$, for redshifted (i.e. positive) velocities, corresponding to the front side of the cloud core, than for blueshifted (i.e. negative) velocities, corresponding to the back side of the core (see Fig. 8). For illustration purposes, we here assume a spherical cloud core in which both the density and the infall velocity increase smoothly toward the center. These are reasonable assumptions for a gravity-dominated, prestellar or protostellar core. We further assume purely radial motions, with an infall velocity profile of the form $v(r) \propto r^{-\alpha}$, with $\alpha>0$ (Fig. 8 shows the case $\alpha=0.5$, which corresponds to free-fall onto a point mass). Then, the loci of constant projected velocity $V_{\text {proj }}$ are closed oval curves which intersect each line of sight at two points, $R_{1}$ and $R_{2}$ for $V_{\text {proj }}>0, B_{1}$ and $B_{2}$ for $V_{\text {proj }}<0$. Denoting the excitation temperature and optical depth at $B_{1}$ and $R_{1}$ by $T_{1}$ and $\tau_{1}$, respectively, and the corresponding quantities at $B_{2}$ and $R_{2}$ by $T_{2}$ and $\tau_{2}$, the line radiation temperature measured in an ON-OFF observation is approximately $T_{B}=J_{\nu}\left(T_{1}\right)\left(1-e^{-\tau_{1}}\right) e^{-\tau_{2}}+J_{\nu}\left(T_{2}\right)\left(1-e^{-\tau_{2}}\right)-J_{\nu}\left(T_{b g}\right)\left(1-e^{-\left(\tau_{1}+\tau_{2}\right)}\right)$ on 


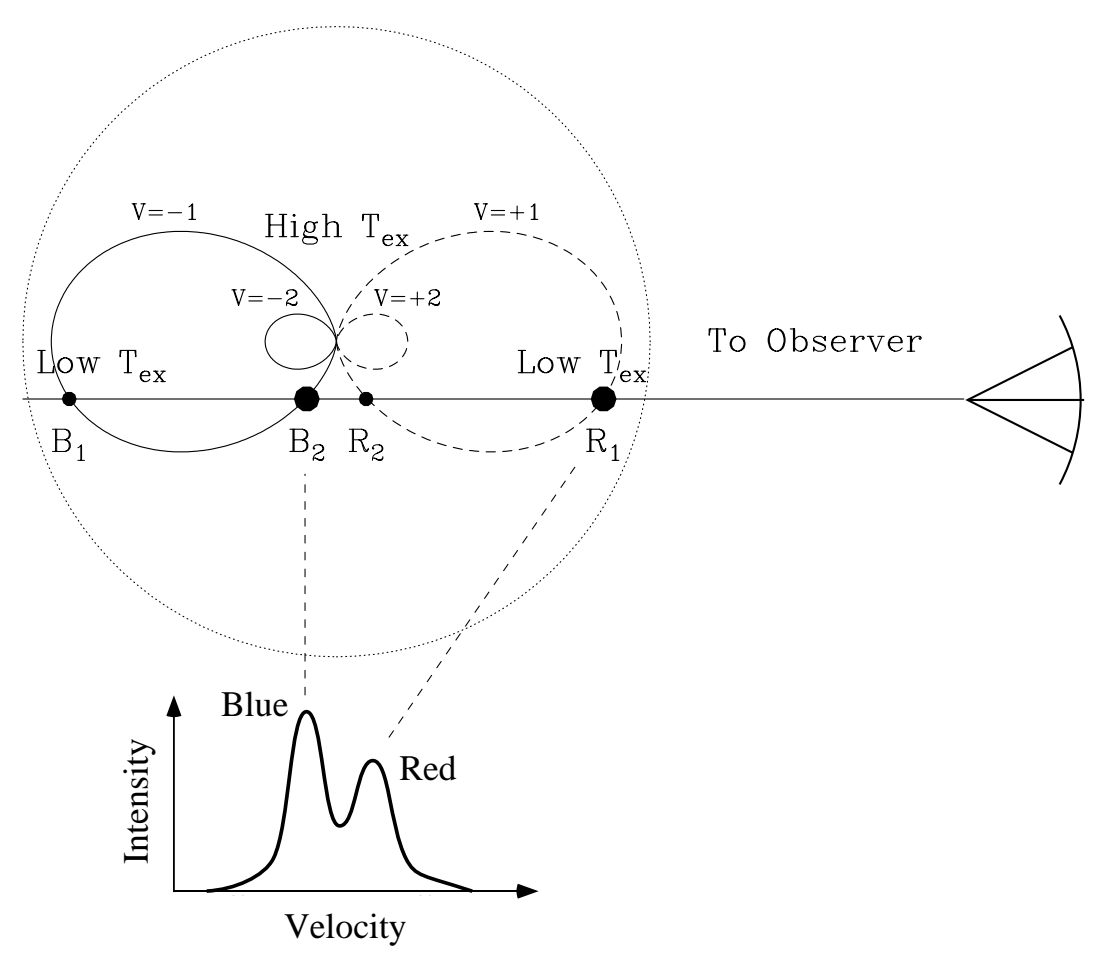

Figure 8: A schematic explanation of why optically thick lines have self-absorbed profiles with a stronger blue peak in a centrally-condensed cloud core with inward motions. The ovals in the upper part of the figure mark loci of constant line-of-sight velocity (here assuming $v(r) \propto r^{-0.5}$ ). For a given velocity, each line of sight intersects these loci at two points, but an opaque transition 'sees' only the point closer to the observer $\left(\mathrm{R}_{1}\right.$ on the red side and $\mathrm{B}_{2}$ on the blue side). As excitation temperatures are higher closer to core center, $T_{e x}\left(B_{2}\right)>T_{e x}\left(R_{1}\right)$, and the blue peak is stronger than the red peak. (Adapted from Zhou \& Evans 1994.)

the blue side, and $T_{R}=J_{\nu}\left(T_{2}\right)\left(1-e^{-\tau_{2}}\right) e^{-\tau_{1}}+J_{\nu}\left(T_{1}\right)\left(1-e^{-\tau_{1}}\right)-J_{\nu}\left(T_{b g}\right)\left(1-e^{-\left(\tau_{1}+\tau_{2}\right)}\right)$ on the red side, where $J_{\nu}(T)=B_{\nu}(T) c^{2} /\left(2 k_{B} \nu^{2}\right)$ (i.e., $J_{\nu}(T) \approx T$ when $B_{\nu}(T)$ is in the Rayleigh-Jeans regime) and $T_{b g} \approx 2.7 \mathrm{~K}$ is the temperature of the cosmic background radiation. Thus, the difference between the blue-shifted and the red-shifted emission does not depend on $T_{b g}$ and is:

$$
T_{B}-T_{R}=\left[J_{\nu}\left(T_{2}\right)-J_{\nu}\left(T_{1}\right)\right]\left(1-e^{-\tau_{1}}\right)\left(1-e^{-\tau_{2}}\right) \approx T_{2}-T_{1}>0
$$

if $\tau_{1}>>1, \tau_{2}>>1$, and $h \nu<<k_{B} T_{i}$.

The infall motions are sometimes difficult to disentangle from the rotation and/or outflow components (e.g. Menten et al. 1987, Cabrit et al. 1996). However, if maps are available in both an optically thick line (e.g., $\mathrm{CS}(2-1)$ or $\mathrm{H}_{2} \mathrm{CO}\left(3_{12}-2_{11}\right)$ ) and the corresponding optically thin tracer (e.g., $\mathrm{C}^{34} \mathrm{~S}(2-1)$ ), the former showing the characteristic collapse asymmetry and the latter being symmetric and peaking at the absorption dip of the other, then the infall interpretation is reasonably secure (e.g. Zhou \& Evans 1994).

Based on this spectroscopic signature, a number of infall surveys have been carried out in recent years. Mardones et al. (1997) observed a sample of 47 embedded YSOs in $\mathrm{H}_{2} \mathrm{CO}\left(2_{12}-1_{11}\right)$ and $\mathrm{CS}(2-1)$, and found more prominent infall in Class 0 than in Class I sources: In these transitions, infall asymmetries are detected toward $40-60 \%$ of Class 0 objects but less than $10 \%$ of Class I sources. This is qualitatively consistent with a decline of infall/accretion rate with evolutionary stage (see $\S 3.5$ below). However, a more recent survey by Gregersen et al. (2000) using $\mathrm{HCO}^{+}(3-2)$ found "infall profiles" in up to $\sim 50 \%$ of Class I sources. The $\mathrm{HCO}^{+}(3-2)$ line is more optically thick than $\mathrm{H}_{2} \mathrm{CO}\left(2_{12}-1_{11}\right)$ and $\mathrm{CS}(2-1)$, hence a better tracer of infall at advanced stages. The latter result shows that some infall is still present at the Class I stage but remains consistent with a decline of the net accretion rate with time. The outflow is so broad in Class I sources that there often 
appears to be little transfer of mass to the inner $\sim 2000 \mathrm{AU}$ radius region around these objects (e.g. Fuller et al. 1995, Cabrit et al. 1996, Brown \& Chandler 1999).

The infall signature has also been detected toward prestellar cores. Lee, Myers, \& Tafalla (1999) observed the central positions of 220 starless cores, detected CS(2-1) and $\mathrm{N}_{2} \mathrm{H}^{+}(1-0)$ in 69 of them, and found strong/probable indication of infall motions in $7 / 17$ of those (i.e., 10-25\%). A follow-up mapping study in the same molecular tracers (Lee, Myers, \& Tafalla 2001) confirmed the presence of infall in most of the single-pointing infall candidates and showed that the inward motions were generally extended (see also below). Interestingly, using $\mathrm{HCO}^{+}(3-2)$, Gregersen \& Evans (2000) found infall asymmetry in up to $50 \%$ of the prestellar cores detected in the submillimeter continuum by WardThompson et al. (1994), which all have $\mathrm{N}_{c e n} \gtrsim 2 \times 10^{22} \mathrm{~cm}^{-2}$. Thus, the presence of inward motions appears to be more likely in more centrally-condensed starless cores, closer to protostar formation.

To go further and turn the qualitative signature of collapse into a quantitative diagnostic of the infall velocity field, both comprehensive mapping and radiative transfer modeling are required. Only a few Class 0 sources and prestellar cores have been studied in detail up to now: B335 (e.g. Choi et al. 1995), L1527 (e.g. Zhou et al. 1996), IRAS 16293 (e.g. Narayanan et al. 1998), IRAM 04191 (e.g. Belloche et al. 2001b), and L1544 (Tafalla et al. 1998, Williams et al. 1999, Ohashi et al. 1999). The observed inward motions are typically spatially extended over $\sim 0.03-0.1 \mathrm{pc}$ and, in the case of prestellar cores, subsonic ( $\sim 0.02-0.1 \mathrm{~km} \mathrm{~s}^{-1}-$ e.g., Tafalla 2001). Evidence for the presence of supersonic and/or turbulent infall speeds ( $\gtrsim 0.3-0.5 \mathrm{~km} \mathrm{~s}^{-1}$ ) is found toward at least some protostars (e.g. Mardones et al. 1997, Di Francesco et al. 2001).

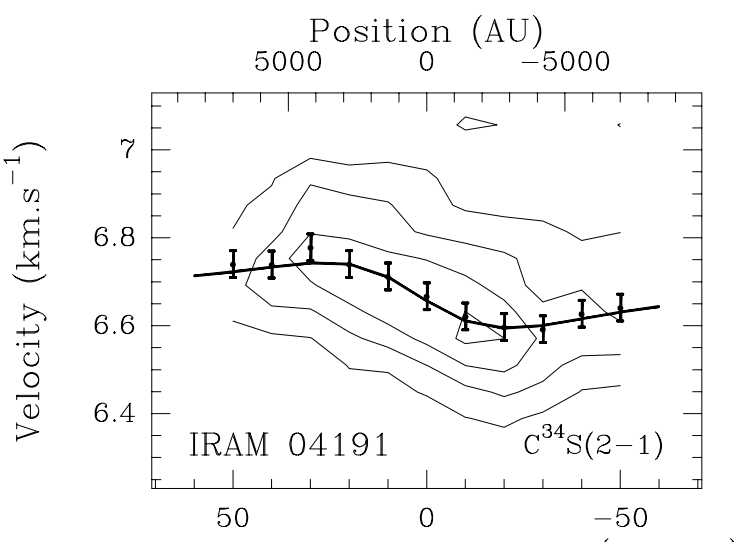

Position along major axis (arcsec)

Figure 9: Position-velocity diagram along the major axis of the IRAM 04191 envelope (i.e., perpendicular to the outflow) derived from a $\mathrm{C}^{34} \mathrm{~S}(2-1)$ map with the $30 \mathrm{~m}$ telescope. Contours: 0.2 to 0.8 by $0.2 \mathrm{~K}$. The dots with error bars mark the observed velocity centroids. Note the strong velocity gradient, indicative of rotation. The solid curve corresponds to a model with differential rotation $\left(V_{\text {rot }}(r) \propto r^{0.3 \pm 0.7}\right.$ for $r<3500 \mathrm{AU}$ and $V_{\text {rot }}(r) \propto r^{-1.5}$ for $\left.r>3500 \mathrm{AU}\right)$ also used in Fig. 7 (see Belloche et al. 2001b).

\subsection{Rotation}

The angular momentum of prestellar cores is an important parameter which controls the possibility of fragmentation during collapse ( $\mathrm{cf} . \oint 3.3 .1$ ) and the formation of any rotationally-supported disk during the protostellar phase (cf. § 3.3.2). Numerical models 
are now beginning to be able to follow the evolution of the angular momentum distribution during collapse (e.g. Tomisaka 2000).

Observationally, rotation about any axis other than the line of sight leads to spatially continuous gradients of radial velocity in the plane of the sky. Once the direction of the velocity gradient has been determined (through, e.g., planar least-squares fitting - see Goodman et al. 1993), the level of projected rotation is best estimated by taking positionvelocity cuts along that direction in strong, optically thin (or only moderately thick) line tracers (see example in Fig. 9). Following the pioneering work of Arquilla \& Goldsmith (1986), Goodman et al. (1993) and Kane \& Clemens (1997) searched for rotation on scales of $\sim 0.1 \mathrm{pc}$ in 43 cloud cores and 15 starless globules using the $(J, K)=(1,1)$ transition of $\mathrm{NH}_{3}$ and $J=2 \rightarrow 1$ transition of ${ }^{13} \mathrm{CO}$, respectively. In most cores and globules, they found small but significant gradients, ranging from 0.3 to $4 \mathrm{~km} \mathrm{~s}^{-1} \mathrm{pc}^{-1}$, which corresponds to $\Omega_{\text {rot }} \sim 10^{-14}-10^{-13} \mathrm{rad} \mathrm{s}^{-1}$ (before any deprojection). On such scales, rotation is thus not energetically dominant in the support of cores since the ratio of rotational to gravitational energy is typically $\beta \sim 0.02$ (e.g. Goodman et al. 1993). The corresponding values of the specific angular momentum $j \equiv J / M\left(\sim 10^{21}-10^{22} \mathrm{~cm}^{2} \mathrm{~s}^{-1}\right)$ are four orders of magnitude larger than the specific angular momentum of the present-day solar system $\left(j_{\odot} \sim 2 \times 10^{17} \mathrm{~cm}^{2} \mathrm{~s}^{-1}\right)$, which is one expression of the "angular momentum problem of star formation" (see also Tomisaka 2000). Interestingly, however, the distribution of core specific angular momenta largely overlaps the $J / M$ distribution measured for the PMS binary systems of Taurus (Simon et al. 1995). This suggests that the high frequency of binary stars (e.g. Duquennoy \& Mayor 1991, Ghez et al. 1993) may result from the rotation of cloud cores, which may "solve their angular momentum problem" by fragmenting into binaries as they collapse.

\subsubsection{Protobinaries}

In agreement with this view, many Class 0 protostars are observed to be multiple systems, when viewed at sub-arcsecond resolution, sharing a common envelope and sometimes a circumbinary disk (e.g. Looney et al. 2000 and Mundy, Looney, \& Welch 2000). Most of these protobinaries probably formed by dynamical fragmentation during (or at the end of) the isothermal collapse phase (e.g. Boss \& Myhill 1995, Bonnell 1999, Bate 2000). Interestingly, only moderately centrally-condensed cores, with inner density profiles as flat as $\rho \propto r^{-1}$ or flatter (like observed prestellar cores - see $\S 2.1$ ), are apparently able to fragment during collapse (Myhill \& Kaula 1992, Burkert et al. 1997). Very close (common-disk) protobinaries with separations of order or less than a few tens of AU, such as L1551-IRS5 (e.g. Rodríguez et al. 1998), point to the process of rotationally-driven disk fragmentation (cf. Bonnell 1999).

\subsubsection{Circumstellar disks}

During the protostellar accretion phase, the size of the rotationally-supported disk is given by the centrifugal radius, $R_{C}$, which defines the position where the centrifugal force balances gravity in the equatorial plane. Most collapse models predict that $R_{C}$ should increase with time as material of higher and higher angular momentum falls in, but the exact dependence on time $t$, or alternatively accumulated central mass $m$, varies from model to model. For instance, if at point mass formation $(t=0)$ the collapsing cloud core is well described by a non-magnetized singular isothermal sphere in (relatively slow) solid-body rotation, $R_{C}$ is predicted to be a steep function of $m$ or $t$ (Terebey, Shu, Cassen 1984):

$$
R_{C} \approx 39 \mathrm{AU}\left(\frac{\Omega}{10^{-14} \mathrm{rad} \mathrm{s}^{-1}}\right)^{2}\left(\frac{a}{0.2 \mathrm{~km} \mathrm{~s}^{-1}}\right)^{-8}\left(\frac{m}{1 M_{\odot}}\right)^{3}
$$


where $m \approx\left(a^{3} / G\right) t$ and $\Omega$ is the initial (or background) cloud rotation rate.

In contrast, a magnetically-controlled, dynamical cloud collapse yields a strong differential rotation $\Omega \propto 1 / r$ at $t=0$, which in turn implies a shallower, linear dependence of $R_{C}$ on $m$ (cf. Basu 1998):

$$
R_{C} \approx 15 \mathrm{AU}\left(\frac{\Omega}{10^{-14} \mathrm{rad} \mathrm{s}^{-1}}\right)^{2}\left(\frac{B}{30 \mu G}\right)^{-2}\left(\frac{m}{1 M_{\odot}}\right)
$$

where $B$ is the magnetic field strength in the background cloud. (Note that, in this case, the accretion rate is not constant with time: It peaks around $\sim 10 \times a^{3} / G$ at $t \sim 0$ and gradually decreases to $\sim a^{3} / G$ thereafter - see $\S 4$ below.)

Observationally, it is quite difficult to discriminate between the disk and envelope components in deeply embedded protostars. Nevertheless, recent (sub)millimeter continuum interferometric measurements have managed to detect compact $(\lesssim 100 \mathrm{AU}$ ), disk-like structures toward several low-mass Class 0/Class I objects (e.g. Chandler et al. 1995, Pudritz et al. 1996, Motte 1998, Looney et al. 2000). The "disks" of Class 0 objects are a factor of $\gtrsim 10$ less massive than their surrounding circumstellar envelopes. By contrast, the disk is a very significant (sometimes dominant) component in more evolved (Class I and Class II) sources (e.g. Lay et al. 1994). Overall, current interferometric results are roughly consistent with the idea that the accretion disk grows from the Class 0 to the Class I stage and achieves its full, protoplanetary size at the Class II/T Tauri stage. The detailed manner in which this growth in size and mass occurs with time is, however, unclear at present. Much progress is expected in this area from future large interferometers operating at near-IR and submillimeter wavelengths such as the VLTI (e.g. Malbet 2001) and ALMA (e.g. Wootten 2001).

\subsection{Outflow}

Most, if not all, Class 0 protostars drive powerful, "jet-like" CO molecular outflows (see, e.g., Bachiller 1996, Richer et al. 2000, and Cabrit, this volume for reviews). The mechanical luminosities of these outflows are often of the same order as the bolometric luminosities of the central sources (e.g. AWB93, Barsony et al. 1998). In contrast, while there is good evidence that some outflow activity exists throughout the accretion phase (e.g. Parker et al. 1991, Bontemps et al. 1996), the CO outflows from Class I sources tend to be much less powerful and less collimated than those from Class 0 objects.

In an effort to quantify the evolution of mass loss during the protostellar phase, Bontemps et al. (1996 - hereafter BATC) obtained and analyzed a homogeneous set of $\mathrm{CO}(2-1)$ outflow data around a large sample of low-luminosity $\left(L_{b o l}<50 L_{\odot}\right)$, nearby $(d<450 \mathrm{pc})$ self-embedded YSOs. Their results show that Class 0 objects lie an order of magnitude above the well-known (e.g. Cabrit \& Bertout 1992) correlation between outflow momentum flux $\left(F_{\mathrm{CO}}\right)$ and bolometric luminosity $\left(L_{\mathrm{bol}}\right)$ holding for Class I sources (see Fig. 5 of BATC). Furthermore, as shown in Fig. 10, BATC found that $F_{\mathrm{CO}}$ was well correlated with $M_{e n v}$ in their entire sample (including both Class I and Class 0 sources). The same correlation was noted independently on other source samples by Moriarty-Schieven et al. (1994), Hogerheijde et al. (1998), and Henning \& Launhardt (1998). As argued by BATC, this new correlation is independent of the $F_{\mathrm{CO}}-L_{\text {bol }}$ correlation and most likely results from a progressive decrease of outflow power with time during the accretion phase. This is illustrated in the normalized $F_{\mathrm{CO}} \mathrm{c} / \mathrm{L}_{\mathrm{bol}}$ versus $M_{\mathrm{env}} / L_{\mathrm{bol}}^{0.6}$ diagram of Fig. 12 below, which should be essentially free of any luminosity effect. 


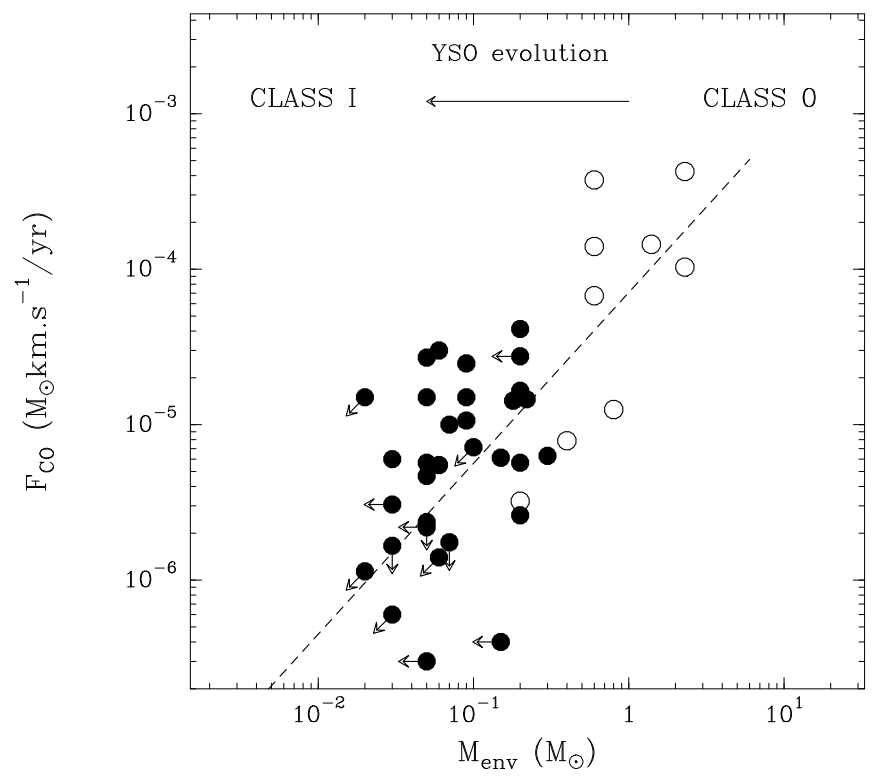

Figure 10: Outflow momentum flux $F_{\mathrm{CO}}$ versus circumstellar envelope mass $M_{\text {env }}$ for a sample of nearby Class I (filled circles) and Class 0 (open circles) YSOs (Bontemps et al. 1996). (The arrows on the data points indicate upper limits.) The 'best fit' $F_{\mathrm{CO}}-M_{\mathrm{env}}$ correlation is plotted as a dashed line. This diagram shows a clear decline of outflow power from Class 0s to Class Is.

\subsection{Time evolution of the accretion/ejection rate}

The mass accretion rate during the protostellar phase is an important characteristic which sets strong constraints on possible collapse models (see, e.g, $\S 4$ below). Measuring the infall velocity and $\dot{M}_{\text {acc }}$ directly is observationally difficult and currently impossible for a large sample of objects (cf. $\S 3.2$ ). One may nevertheless use outflow measurements, which are much easier, to obtain indirect clues to the accretion history of protostars. Magnetocentrifugal accretion/ejection models of bipolar outflows (e.g. Ferreira \& Pelletier 1995, Fiege \& Henriksen 1996, Königl \& Pudritz 2000, Shu et al. 2000) indeed predict a direct proportionality between accretion and ejection. On this basis, it has been proposed that the decline of outflow/jet power with evolutionary stage seen in Fig. 10 and Fig. 12 reflects a corresponding decrease in the mass-accretion/infall rate (Bontemps et al. 1996 - BATC). The results of BATC indicate that $\dot{M}_{\text {jet }}$ declines from $\sim 10^{-6} \mathrm{M}_{\odot} \mathrm{yr}^{-1}$ for the youngest Class 0 protostars to $\sim 2 \times 10^{-8} \mathrm{M}_{\odot} \mathrm{yr}^{-1}$ for the most evolved Class I sources, suggesting a decrease in $\dot{M}_{\text {acc }}$ from $\sim 10^{-5} \mathrm{M}_{\odot} \mathrm{yr}^{-1}$ to $\sim 2 \times 10^{-7} \mathrm{M}_{\odot} \mathrm{yr}^{-1}$ if realistic jet model parameters are adopted $\left(\dot{M}_{\text {jet }} / \dot{M}_{\text {acc }} \sim 0.1-0.3, V_{\text {jet }} \sim 100 \mathrm{~km} \mathrm{~s}^{-1}\right)$. These values of $\dot{M}_{\text {acc }}$ for Class 0 and Class I protostars agree well with independent estimates of the rates of envelope dissipation based on circumstellar mass versus age arguments (cf. Ladd, Fuller, \& Deane 1998).

Note that the decline of outflow power from Class 0 to Class I objects is more pronounced in star-forming clusters, such as the $\rho$ Ophiuchi main cloud, than in the Taurus cloud where stars form in relative isolation (Henriksen, André, \& Bontemps 1997 - cf. Fig. 12). In Ophiuchus and Perseus, the outflow data suggest that $\dot{M}_{\text {acc }}$ could even reach $\gtrsim 10 \times$ $a^{3} / G$ or $\sim 10^{-4} \mathrm{M}_{\odot} \mathrm{yr}^{-1}$ in the most extreme Class 0 objects. Interestingly, such high values of $\dot{M}_{\text {acc }}$ have also been inferred for some Class 0 sources based on independent SED modeling (Jayawardhana, Hartmann, \& Calvet 2001). High accretion rates at the Class 0 stage are also in line with the large envelope densities measured for these objects in protoclusters (Motte \& André 2001 - see $\S 2.2$ above).

Very few direct estimates of $\dot{M}_{\text {acc }}$ exist at this time but they do seem to support 
the conclusions inferred from outflow studies. While moderate accretion rates $(\sim 1-$ $5 \times 10^{-6} \mathrm{M}_{\odot} \mathrm{yr}^{-1}$ ) have been found for several Class I sources (e.g. Ohashi 1999), significantly larger accretion rates $\left(\gtrsim 10^{-5} \mathrm{M}_{\odot} \mathrm{yr}^{-1}\right)$ have been reported for some Class 0 objects in Perseus and Ophiuchus (e.g. Ward-Thompson et al. 1996, Ceccarelli et al. 2000, Di Francesco et al. 2001).

\section{Comparison with collapse models}

\subsection{Model predictions}

Whitworth \& Summers (1985) have shown that there is a two-dimensional continuum of self-similar solutions to the problem of isothermal spherical collapse (see also Whitworth 2001). In this continuum, the well-known solutions of Shu (1977) and Larson-Penston (e.g. Larson 1969) represent two extreme limits. All isothermal similarity solutions share a universal evolutionary pattern. At early times $(t<0)$, a compression wave (initiated by, e.g., an external disturbance) propagates inward at the sound speed, leaving behind it a $\rho(r) \propto r^{-2}$ density profile. At $t=0$, the compression wave reaches the center and a point mass forms which subsequently grows by accretion at a constant rate. At later times $(t>$ 0 ), this wave is reflected into a rarefaction or expansion wave, propagating outward (also at the sound speed) through the infalling gas, and leaving behind it a free-fall $\rho(r) \propto r^{-1.5}$ density distribution. The various solutions can be distinguished by the absolute values of the density and velocity around $t \sim 0$. In particular, the Shu (1977) solution, which underlies the 'standard' model of low-mass star formation (Shu et al. 1987), corresponds to a static $(v=0)$ singular isothermal sphere (SIS) with $\rho(r)=\left(a^{2} / 2 \pi G\right) r^{-2}$ at $t=0$ (where $a$ is the isothermal sound speed). At this instant, the Larson-Penston solution is $\sim 4.4$ times denser and far from equilibrium $(v \approx-3.3 a)$. During the accretion phase $(t>0)$, the infall envelope is expected to be a factor $\sim 7$ denser in the Larson-Penston solution. Accordingly, the accretion rate is also much larger in the Larson-Penston case $\left(\sim 47 a^{3} / G\right)$ than in the Shu case $\left(\sim a^{3} / G\right)$.

In practice, however, the initial conditions for fast protostellar collapse are unlikely to be self-similar, and the above similarity solutions can only be taken as plausible asymptotes. Indeed, numerical (magneto)hydrodynamic simulations predict a time-dependent accretion history when the radial density profile at the onset of fast protostellar collapse differs from $\rho \propto r^{-2}$ (e.g. Foster \& Chevalier 1993 - FC93; Safier, McKee, \& Stahler 1997; Li 1998; Ciolek \& Königl 1998). In particular, when starting from a Bonnor-Ebert-like initial density profile with a flat inner plateau as observed in prestellar cores (see $\S 2.1$ ), these collapse simulations find that supersonic infall velocities quickly develop near the center, prior to the formation of the central hydrostatic protostellar object at $t=0$ (e.g. FC93 - see Fig. 11a). Furthermore, it appears that the Larson-Penston similarity solution is generally a good approximation near point-mass formation $t=0$ (at least for small radii), but that the Shu solution is more adequate at intermediate $t \geq 0$ times, before the expansion wave reaches the edge of the initial, pre-collapse dense core.

During the protostellar accretion phase $(t>0)$, because of the significant infall velocities achieved at $t<0, \dot{M}_{\text {acc }}$ is initially higher (by a factor $\lesssim 10$ ) than the standard $\sim a^{3} / G$ value obtained for the inside-out collapse of a static SIS (see Fig. 11c \& Fig. 11d). The accretion rate then converges toward the standard value of the Shu solution, and declines again below $a^{3} / G$ at late times if the reservoir of mass is finite (e.g. Fig. 11).

\subsection{Free-fall approximation}

Because the inflow becomes supersonic early on (at small radii), one may obtain a qualitatively correct description of the collapse and subsequent accretion phase through simple, 

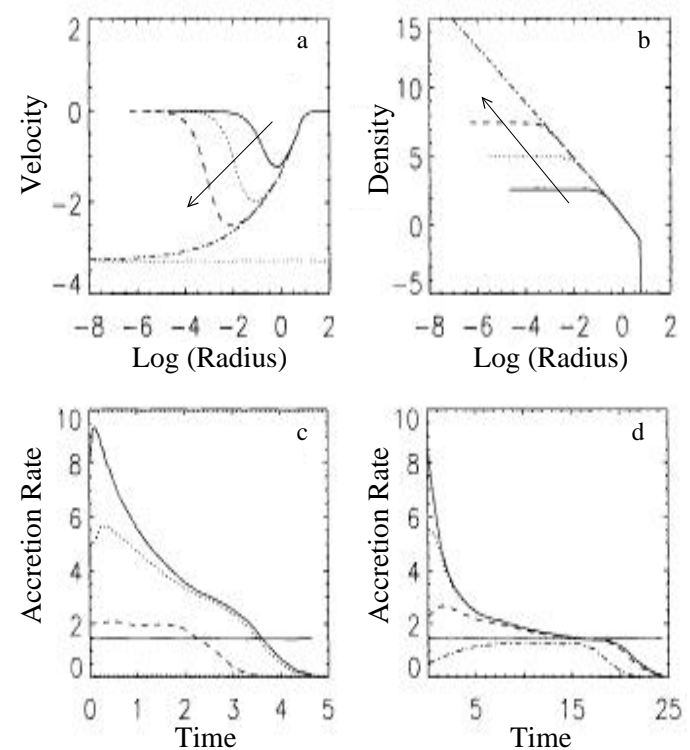

Figure 11: Velocity profiles (a) (in units of the sound speed $a$ ) and density profiles (b) (in units of the initial central density) at several times $(t<0)$ prior to point mass formation according to numerical simulations of the isothermal collapse of a Bonnor-Ebert sphere. The bottom panels show the mass accretion rate $\dot{M}_{\text {acc }}$ (in units of $a^{3} / G$ ) as a function of time (in units of the initial free-fall time) after point mass formation $(t>0)$, when the initial sphere is such that $R_{\text {out }} / R_{\text {flat }}=6.4$ (c) (i.e., critically stable) and $R_{\text {out }} / R_{\text {flat }}=10(\mathrm{~d})$, respectively. (From Foster \& Chevalier 1993.)

pressure-free (i.e. free-fall) analytic calculations (e.g. Henriksen, André, Bontemps 1997 - HAB97). Spherically-symmetric free-fall collapse is best treated using a Lagrangian formalism (e.g. Henriksen 1989). When pressure effects are neglected, the collapse history is indeed entirely determined by the form of the mass distribution, $M(R) \equiv M(r<R)$, at the onset of collapse. In particular, the gravitational acceleration of the shell of initial radius $R$ is simply $-G M(R) / r^{2}$, where only the instantaneous position $r$ of the shell (but not $M(R)$ ) varies with time. It is thus convenient to label each shell using the independent variable $M$ and to invert $M(R)$ into $R(M)$. The free-fall time of the shell is

$$
t_{f f}(M)=\frac{\pi}{2} \sqrt{\frac{R^{3}(M)}{2 G M}},
$$

from which $\frac{d t_{f f}}{d M}$ is easily obtained. At $t-t_{0}=t_{f f}(M)$ (the offset $t_{o}$, which marks the onset of collapse, is introduced so that point mass formation occurs at $t=0$ as usually adopted), the mass infall rate at the center is then simply: $\dot{M}_{a c c}=\left[\frac{d t_{f f}}{d M}\right]^{-1}$. The normalized position $s=r / R$ at time $t$ of the shell initially at $R(M)$ is given by the equation of free-fall from rest:

$$
\frac{t-t_{o}}{t_{f f}(M)}=\frac{2}{\pi}[\operatorname{Arccos}(\sqrt{s})+\sqrt{s(1-s)}] .
$$

This effectively provides the mass distribution $M(r, t)$, and thus the density profile, at time $t$ (cf. Whitworth \& Ward-Thompson 2001). The velocity profile is also easily derived from $\partial r / \partial t(M)=R(M) \partial s / \partial t(M)$. In the end, one obtains semi-analytic, parameterized representations of the mass infall rate and of the density and velocity profiles as a function of time, for any initial density profile. Two examples of plausible infall rate histories calculated following this approach are shown in Fig. 12 (see also below). 
The simple pressure-free aproximation outlined above can help understand the origin of the time dependence of $\dot{M}_{\text {acc }}$. If the initial density profile is strictly flat for $R<R_{\text {flat }}$, then all the shells initially at $R<R_{\text {flat }}$ have the same free-fall time $t_{f f}^{\text {flat }}=\sqrt{3 \pi /\left(32 G \rho_{\text {flat }}\right)}$ by Eq. (10), i.e., the collapse of the central plateau region is homologous, which leads to a formally infinite mass infall rate at $t-t_{o}=t_{f f}^{f l a t}$. In practice, the density is not strictly uniform at $t=t_{o}$ in the central region and pressure gradients develop, which both tend to reduce the strength of the $\dot{M}_{\text {acc }}$ peak at $t=0$. From Eq. (10), one can also see why an $\rho \propto R^{-2}$ initial density profile (i.e., $M \propto R$ ) implies a constant accretion rate at $t>0$ (since $t_{f f}(M) \propto M$ in this case). More generally, if $\rho \propto R^{-p}$ with $0<p<3$ initially, then $t_{f f}(M) \propto M^{p /(6-2 p)}$ and $\dot{M}_{\text {acc }} \propto t^{(6-3 p) / p}$, hence $\dot{M}_{\text {acc }}$ decreases with time for $p>2$ and increases with time for $p<2$.

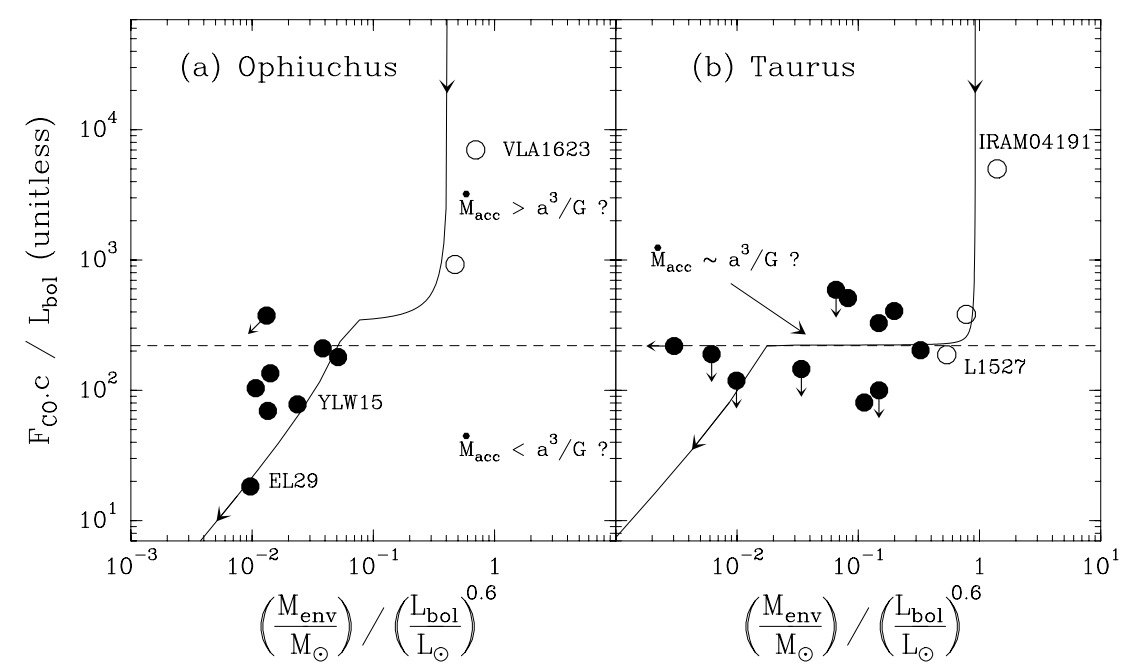

Figure 12: Normalized outflow momentum flux, $F_{\mathrm{CO}} \mathrm{c} / \mathrm{L}_{\mathrm{bol}}$, versus normalized envelope mass, $M_{\text {env }} / L_{\mathrm{bol}}^{0.6}$, for Class 0 (open circles) and Class I (filled circles) objects in Ophiuchus (a) and Taurus (b) (from Bontemps et al. 1996 and Motte \& André 2001). $F_{\mathrm{CO}}$ c/L bol can be taken as an empirical tracer of the accretion rate $\dot{M}_{\text {acc }}$, while $M_{\text {env }} / L_{\mathrm{bol}}^{0.6}$ is an evolutionary indicator which decreases with time. This diagram should therefore mainly reflect the evolution of $\dot{M}_{\text {acc }}$ during the protostellar phase. The solid curves show illustrative accretion rate histories predicted by the simplified collapse model of Henriksen et al. (1997) when the relative mass in the flat inner region of the initial density profile is large (a) and small (b).

\subsection{Models vs. observations}

The apparent decay of $\dot{M}_{\text {acc }}$ from the Class 0 to the Class I stage ( $\left.\S 3.5\right)$ is thus likely a direct consequence of the density structure observed in prestellar cores/condensations ( $\S 2.1)$. Furthermore, by comparison with the rough estimates of $\dot{M}_{\text {acc }}$ given in $\S 3.5$ above, it is tempting to identify the short period of energetic accretion $\left(\dot{M}_{\mathrm{acc}} \sim 10 \times a^{3} / G\right)$ predicted by the models just after point-mass formation with the observationally-defined Class 0 stage (HAB97, see Fig. 12). In this view, the more evolved Class I objects would correspond to the longer period of moderate accretion/ejection when the accretion rate approaches the standard value $\left(\dot{M}_{\text {acc }} \lesssim a^{3} / G \sim 2 \times 10^{-6} \mathrm{M}_{\odot} \mathrm{yr}^{-1}\right.$ for a cloud temperature of $\sim 10 \mathrm{~K}$ ).

Using the simplified pressure-free approach outlined in $\S 4.2$, HAB97 could indeed find a good overall fit to the empirical accretion history inferred by BATC on the basis of 
CO outflow observations (see Fig. 12). Although the detailed predictions of pressure-free models are inconsistent with the small infall velocities observed toward isolated prestellar cores and protostellar envelopes (cf. $\S 3.2$ ), they may be essentially valid in the case of the more violent protostellar collapse occurring in clusters.

In the absence of magnetic fields, Foster \& Chevalier (1993) have shown that the timescale for convergence to the standard accretion rate of Shu (1977) depends on the radius of the flat inner core, $R_{\text {flat }}$, relative to the outer radius of the initial pre-collapse condensation, $R_{\text {out }}$ (see Fig. 11). They found that a phase of constant $\sim a^{3} / G$ accretion rate is achieved only when $R_{\text {out }} / R_{\text {flat }} \gtrsim 20$, typically after $\sim 10$ free-fall times of the flat inner region, for a period lasting $\sim 15$ free-fall times when $R_{\text {out }} / R_{\text {flat }}=20$ and progressively longer as $R_{\text {out }} / R_{\text {flat }}$ increases. Since observations suggest $R_{\text {out }} / R_{\text {flat }} \lesssim 3$ in the Ophiuchus main cloud (Motte et al. 1998) and $R_{\text {out }} / R_{\text {flat }} \gtrsim 15$ in Taurus (see $\S 2.1$ ), one may expect a marked time-dependence of $\dot{M}_{\text {acc }}$ in Ophiuchus but a reasonable agreement with the constant accretion rate of the self-similar theory of Shu et al. $(1987,1993)$ in Taurus. Indeed, HAB97 note that there is a much better continuity between Class 0 and Class I protostars in Taurus than in Ophiuchus (compare Fig. 12a and Fig. 12b).

\section{Global aspects: Origin of the IMF}

On a more global level, the wide-field imaging capabilities offered by current bolometer arrays at (sub)millimeter wavelengths now allow global surveys for both pre-collapse condensations and young protostars to be taken over the whole spatial extent of star-forming clouds (see, e.g., Fig. 13). Such surveys are a powerful tool to gain insight into the origin of stellar masses and the nature of the fragmentation process in the ISM, for which we still have no satisfactory theory (e.g. Elmegreen 2001). Sensitive submillimeter dust emission maps indeed have the remarkable property that they can probe cloud structure, pre-collapse condensations, collapsing/accreting protostars, and post-collapse circumstellar envelopes/disks, simultaneously. They thus make it possible to investigate the genetic link between prestellar condensations and protostars.

This point is illustrated by recent ground-based dust continuum studies around $1 \mathrm{~mm}$, which have found prestellar mass spectra consistent with the stellar IMF.

Using the MPIfR bolometer array on the IRAM $30 \mathrm{~m}$ telescope, Motte, André, \& Neri $\left(1998-\right.$ MAN98) completed a $\sim 480 \operatorname{arcmin}^{2}$ mosaic of the $\rho$ Oph main cloud $(d \approx 150 \mathrm{pc})$ at $1.3 \mathrm{~mm}$. Based on a multi-resolution wavelet analysis, they could identify a total of 100 compact 'condensations' with characteristic angular scales of $\sim 15^{\prime \prime}-30^{\prime \prime}$ (i.e., $\sim 2500-$ $5000 \mathrm{AU})$ in their mosaic. These small-scale condensations consist of 59 starless fragments (undetected by ISOCAM in the mid-IR - cf. Bontemps et al. 2001) and 41 circumstellar envelopes/disks around embedded YSOs (detected at IR and/or radio continuum wavelengths). Comparison of the masses derived from the $1.3 \mathrm{~mm}$ continuum with virial masses from molecular-line data indicates that most of the starless fragments are gravitationally bound (with $M_{1.3} / M_{v i r} \gtrsim 0.5$ ) and will form stars in the near future (e.g. Belloche et al. 2001a). The mass distribution of these 59 compact prestellar condensations, complete down to $\sim 0.1 M_{\odot}$, is remarkable in that it mimics the shape of the stellar IMF (see Fig. 14a). In particular, it is very similar in shape to the YSO mass spectrum recently determined down to $\lesssim 0.1 M_{\odot}$ for the Class II sources of the $\rho$ Oph cluster from ISOCAM $7 \mu \mathrm{m}$ and $15 \mu \mathrm{m}$ observations (Bontemps et al. 2001). Both mass spectra show a break at $\sim 0.3-0.5 M_{\odot}$, indicative of a characteristic stellar/prestellar mass of order $\sim 0.3 M_{\odot}$ in $\rho \mathrm{Oph}$, comparable to the typical Jeans mass in the dense $\mathrm{DCO}^{+}$cores of Loren et al. (1990).

The results of MAN98 have been essentially confirmed by an independent $850 \mu \mathrm{m}$ SCUBA survey of the same region with JCMT (Johnstone et al. 2000). 


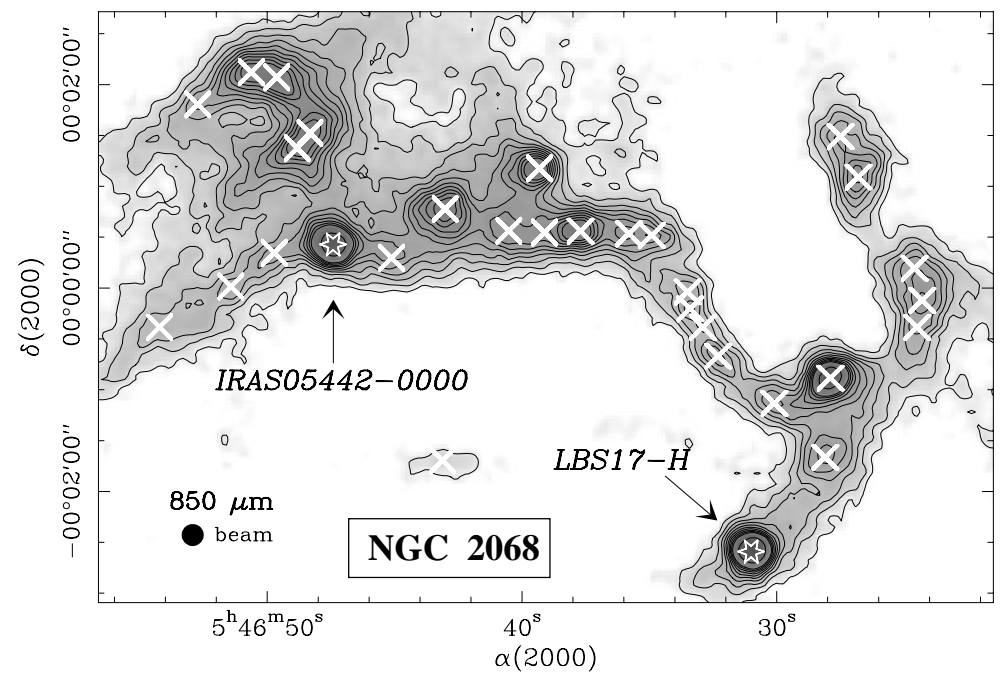

Figure 13: SCUBA $850 \mu \mathrm{m}$ dust continuum map of the NGC 2068 protocluster extracted from the mosaic of NGC 2068/2071 by Motte, André, Ward-Thompson, \& Bontemps (2001). The effective angular resolution is $13^{\prime \prime}$. Contour levels go from 65 to $650 \mathrm{mJy} /$ beam with steps of $65 \mathrm{mJy} /$ beam; last contour is $1 \mathrm{Jy} /$ beam. The mean rms noise level is $\sim 22 \mathrm{mJy} / 13^{\prime \prime}$-beam. A total of 30 starless condensations (cf. crosses) are detected in this $\sim 1 \mathrm{pc} \times 0.7 \mathrm{pc}$ field.

In a related $3 \mathrm{~mm}$ interferometric study with OVRO interferometer, Testi \& Sargent (1998) mosaiced the inner $5.5^{\prime} \times 5.5^{\prime}$ region of the Serpens cloud core, and identified 26 starless condensations with a cumulative mass spectrum $N(>m) \propto m^{-1.1}$ between $\sim 0.5 M_{\odot}$ and $\sim 10 M_{\odot}$, close to the Salpeter IMF.

More recently, Motte et al. (2001 - MAWB) used SCUBA on JCMT to image a $30^{\prime} \times 17^{\prime}$ field at $450 \mu \mathrm{m}$ and $850 \mu \mathrm{m}$ around the NGC 2068/2071 protoclusters in Orion B (see Figs. 13). Their images reveal a total of $\sim 70$ compact starless condensations whose mass spectrum is again reminiscent of the IMF between $\sim 0.6 M_{\odot}$ and $\sim 5 M_{\odot}$ (see Fig. 14b).

Such a resemblance to the IMF suggests that the starless condensations detected in the (sub)millimeter dust continuum on the same spatial scales as protostellar envelopes are the direct progenitors of individual stars or systems. In agreement with this view, some of the condensations show spectroscopic evidence of collapse (Belloche et al. 2001a).

By contrast, note that the typical clump mass spectra $\left(N(>m) \propto m^{-0.5}\right)$ found by largescale $\mathrm{CO}$ studies of molecular clouds are much shallower than the IMF above $\sim 0.5 M_{\odot}$ (e.g. Kramer et al. 1998, Williams et al. 2000). The difference presumably arises because $\mathrm{CO}$ studies are primarily sensitive to transient unbound structures which are not immediately related to star formation.

These recent findings on the mass spectrum of protocluster condensations are very encouraging because they favor the view according to which the low-mass end of the IMF is at least partly determined by turbulent fragmentation at the prestellar stage of star formation (e.g. Larson 1999 and Elmegreen 2001). A plausible scenario, supported by some numerical simulations of cluster formation (Klessen \& Burkert 2000, Padoan et al. 2001), could be the following. First, cloud turbulence generates a field of density fluctuations, a fraction of them corresponding to self-gravitating fragments. Second, these fragments (or "kernels") decouple from their turbulent environment (e.g. Myers 1998) and collapse to protostars after little interaction with their surroundings.

In any event, to understand how the IMF comes about, it appears crucial to investigate the processes by which prestellar condensations form in molecular clouds. 


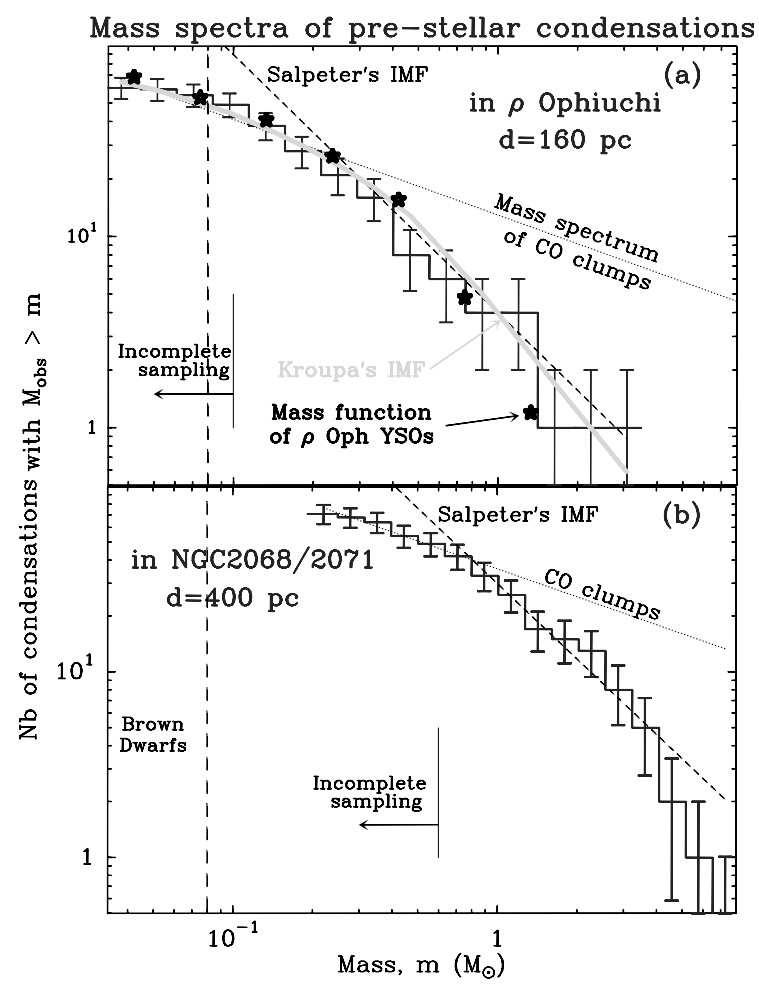

Figure 14: Cumulative mass distributions of the prestellar condensations found by MAN98 and MAWB in the $\rho$ Oph (a) and NGC 2068/2071 (b) protoclusters. The dotted and dashed lines show power-laws of the form $N(>m) \propto m^{-0.5}$ (mass spectra of CO clumps, see Williams et al. 2000) and $N(>m) \propto m^{-1.35}$ (Salpeter's IMF), respectively. The solid curve in (a) shows the shape of the field star IMF (Kroupa et al. 1993), and the star markers represent the mass function of $\rho$ Oph YSOs derived from a mid-IR survey with ISOCAM (Bontemps et al. 2001).

\section{Conclusions and future prospects}

The observational studies summarized in $\S 2.1$ demonstrate that prestellar cores/fragments are characterized by flat inner radial density gradients (e.g. Fig. 4b). This, in turn, suggests the initial conditions for fast protostellar collapse are non-singular, i.e., the density profile at the onset of collapse is not infinitely centrally condensed. (Sub)millimeter observations also set strong constraints on protostellar evolution. The fact that young (Class 0 ) protostars drive more powerful outflows than evolved (Class I) protostars suggests that the mass accretion rate $\dot{M}_{\text {acc }}$ decreases by typically a factor of $\sim 5-10$ from the Class 0 to the Class I stage ( $\S 3.5$ and Fig. 12 ). Such a decline in $\dot{M}_{\text {acc }}$ during the protostellar accretion phase may be the direct result of the form of the initial, pre-collapse density profile (see $\S 4$ ). Based on these observational constraints, we suggest that most protostars form in a dynamical rather than quasi-static fashion.

The results discussed in this paper also have broader implications concerning, e.g., the origin of the IMF. As pointed out in $\S 2.1$ and $\S 2.2$, the prestellar condensations observed in regions of multiple star formation such as $\rho$ Ophiuchi are finite-size structures, typically a few $1000 \mathrm{AU}$ in radius, which are clearly not scale-free. This favors a picture of star formation in clusters in which individual protostellar collapse is initiated in compact condensations resulting from fragmentation and resembling more finite-size Bonnor-Ebert cloudlets than singular isothermal spheres. Such condensations may correspond to dense, low-ionization pockets decoupling themselves from the parent molecular cloud as a result of ambipolar diffusion and/or the dissipation of MHD turbulence (e.g. Mouschovias 1991, Myers 1998, Nakano 1998). They would thus be free to undergo Jeans-like gravitational 
instabilities and collapse under the influence of external disturbances, of which there are many types in regions of multiple star formation (e.g. Whitworth et al. 1996, Motte et al. 1998, Barsony et al. 1998). By contrast, the low-density regions of the ambient cloud, being more ionized, would remain supported against collapse by static and/or turbulent magnetic fields. The typical separation between individual condensations should be of order the Jeans length in the parent cloud/core, in rough agreement with observations (e.g. Motte et al. 1998).

In this observationally-driven scenario of fragmentation and collapse, stars are built from bounded fragments which represent finite reservoirs of mass. The star formation efficiency within these fragments is high: most of their 'initial' masses at the onset of collapse end up in stars. If this is true, it implies that the physical mechanisms responsible for the formation of prestellar cores/condensations in molecular clouds, such as turbulent fragmentation (e.g. Padoan et al. 2001), play a key role in determining the IMF of embedded clusters. Such a picture, which we favor for regions of multiple star formation, is consistent with recent numerical simulations of protocluster formation (e.g. Klessen \& Burkert 2000). In regions of more distributed star formation like the Taurus cloud, however, protostars may accrete from effectively larger reservoirs of mass, and feed-back processes such as stellar winds may be more important in limiting accretion and defining stellar masses (e.g. Shu et al. 1987, Adams \& Fatuzzo 1996, Velusamy \& Langer 1998).

With the advent of major new facilities at far-IR and submillimeter wavelengths, the end of the present decade promises to be rich in observational breakthroughs concerning the earliest stages of star formation. With an angular resolution at 85-300 $\mu \mathrm{m}$ comparable to (or better than) the largest ground-based millimeter radiotelescopes, Herschel/FIRST, the Far InfraRed and Submillimeter Telescope to be launched by ESA in 2007, will make possible deep, unbiased surveys for Class 0 protostars and prestellar condensations in all the nearby $(d \lesssim 1 \mathrm{kpc}$ ) molecular cloud complexes of the Galaxy (cf. Pilbratt et al. 2001 and references therein). These surveys will provide, for the first time, reliable lifetimes for the various protostellar phases, as well as mass and luminosity functions for complete samples of prestellar objects down to substellar masses. The thermal and density structure of these objects will also be accessible with unprecedented accuracy.

High-resolution ( $\lesssim 0.1-1^{\prime \prime}$ ) follow-up observations with the "Atacama Large Millimeter Array' (ALMA, available around 2008 - cf. Wootten 2001) at $\sim 450 \mu \mathrm{m}-8.5 \mathrm{~mm}$ will resolve multiple systems and beat source confusion in protoclusters. This will give a direct estimate of the fraction of protobinaries (cf. Looney, Mundy, \& Welch 2000) and will set strong constraints on viable mechanisms for the formation of binary systems. Detailed studies of the kinematics of protostellar envelopes in a large number of molecular line tracers will also be possible on a routine basis, and will yield direct estimates of the infall, outflow, and rotation rates.

Complementing each other nicely, Herschel and ALMA should tremendously improve our global understanding of the initial stages of star formation in the Galaxy.

\section{References}

Abergel, A., Bernard, J.P., Boulanger, F. et al. 1996, A\&A, 315, L329

Adams F.C., Fatuzzo, M. 1996, ApJ, 464, 256

Adams F.C., Lada C.J., Shu, F.H. 1987, ApJ, 312, 788

Alves, J.F., Lada, C.J., \& Lada, E.A. 2001, Nature, 409, 159

André, P., Montmerle, T. 1994, ApJ, 420, 837 (AM94)

André, P., Motte, F., Bacmann, A. 1999, ApJL, 513, L57

André, P., Ward-Thompson, D., Barsony, M. 1993, ApJ, 406, 122 (AWB93) 
André, P., Ward-Thompson, D., Barsony, M. 2000, in Protostars and Planets IV, eds. V. Mannings, A.P. Boss, \& S.S. Russell (Univ. of Arizona Press, Tucson), p. 59

André, P., Ward-Thompson, D., Motte, F. 1996, A\&A, 314, 625

Arons, J. \& Max, C.E. 1975, ApJ, 196, L77

Arquilla, R., \& Goldsmith, P.F. 1986, ApJ, 303, 356

Bachiller, R. 1996, ARA\&A, 34, 111

Bacmann, A., André, P., Puget, J.-L., Abergel, A., Bontemps, S., \& Ward-Thompson, D. 2000, A\&A, 361, 555

Barrett, A.H., Meeks, M.L., Weinreb, S. 1964, AJ, 69, 134

Barsony, M., Ward-Thompson, D., André, P., O'Linger, J. 1998, ApJ, 509, 733

Basu, S. 1998, ApJ, 509, 229

Basu, S., \& Mouschovias, T.Ch. 1995, ApJ, 453, 271 (BM95)

Bate, M.R. 1998, ApJL, 508, L5

Bate, M.R. 2000, MNRAS, 314, 33

Beckwith, S.V.W., Sargent A.I, Chini, R.S., Guesten, R. 1990, AJ, 99, 924

Beichman, C.A., Myers, P.C., Emerson, J.P. et al. 1986, ApJ, 307, 337

Belloche, A., André, P., \& Motte, F. 2001a, in From Darkness to Light, Eds. T. Montmerle \& P. André, ASP Conf. Ser., 243, p. 313

Belloche, A., André, P., Despois, D., Bacmann, A., \& Motte, F. 2001b, in The Origin of Stars and Planets: The VLT View, Eds. J. Alves \& M. McCaughrean (Springer-Verlag, Berlin), Poster Proceedings of the ESO Workshop (CD-ROM), in press

Benson, P. J., Myers, P. C. 1989, ApJS, 71, 89

Bernard, J.P., Boulanger, F., Puget, J.L. 1993, A\&A, 277, 609

Bertout, C., Basri, G., \& Bouvier, J. 1988, ApJ, 330, 350

Blottiau, P., Chièze, J.P., \& Bouquet, S. 1988, A\&A, 207, 24

Bonnell, I.A. 1999, in The Origin of Stars and Planetary Systems, Eds. C.J. Lada \& N.D. Kylafis (Kluwer, Dordrecht), p. 479

Bonnor, W.B. 1956, MNRAS, 116, 351

Bontemps, S. 1996, PhD thesis, University of Paris XI

Bontemps, S., André, P., Terebey, S., Cabrit, S. 1996, A\&A, 311, 858 (BATC)

Bontemps, S., André, P., Kass, A.A., Nordh, L., Olofsson, G. et al. 2001, A\&A, in press (astro-ph/0103373)

Boss, A.P., Myhill, E.A. 1995, ApJ, 451, 218

Boss, A.P., Yorke, H.W. 1995, ApJL, 439, L55

Brown, D.W., \& Chandler, C.J. 1999, MNRAS, 303, 855

Burkert, A., Bate, M.R., \& Bodenheimer, P. 1997, MNRAS, 289, 497

Cabrit S., \& Bertout C. 1992, A\&A, 261, 274

Cabrit, S., Guilloteau, S., André, P., Bertout, C., Montmerle, T., \& Schuster, K. 1996, A\&A, 305, 527

Caselli, P., \& Myers, P.C. 1995, ApJ, 446, 665

Ceccarelli, C., Castets, A., Caux, E. et al. 2000, A\&A, 355, 1129

Chandler, C.J., \& Richer, J.S. 2000, ApJ, 530, 851

Chandler, C.J., Koerner, D.W., Sargent, A.I., \& Wood, D.O.S 1995, ApJ, 449, L139

Chen, H., Myers, P.C., Ladd, E.F., \& Wood, D.O.S. 1995, ApJ, 445, 377

Choi, M., Evans, N.J., II, Gregersen, E.M., \& Wang, Y. 1995, ApJ, 448, 742

Ciolek, G.E., \& Basu, S. 2001, in From Darkness to Light, Eds. T. Montmerle \& P. André, 
ASP Conf. Ser., 243, p. 79

Ciolek, G.E., \& Königl, A. 1998, ApJ, 504, 257

Ciolek, G.E., Mouschovias, T.Ch. 1995, ApJ, 454, 194 (CM95)

Clemens, D.P., \& Barvainis, R. 1988, ApJS, 68, 257

Crutcher, R.M. 1999, ApJ, 520, 706

Crutcher, R.M., \& Troland, T.H. 2000, ApJ, 537, L139

Curry, C.L., \& McKee, C.F. 2000, ApJ, 528, 734

Di Francesco, J., Myers, P.C., Wilner, D.J. \& Ohashi, N. 2001, ApJ, in press

Draine, B.T., Lee, H.M. 1984, ApJ, 285, 89

Duquennoy, A., \& Mayor, M. 1991, A\&A, 248, 485

Elmegreen, B.G. 1997, ApJ, 486, 944

Elmegreen, B.G. 2001 in From Darkness to Light, Eds. T. Montmerle \& P. André, ASP

Conf. Ser., 243, p. 255

Emerson, D.T., Klein, U., Haslam, C.G.T. 1979, A\&A, 76, 92

Evans, N.J. 1999, ARA\&A, 37, 311

Falgarone, E., \& Pety, J. 2001 in From Darkness to Light, Eds. T. Montmerle \& P. André, ASP Conf. Ser., 243, p. 53

Ferreira J., \& Pelletier G. 1995, A\&A, 295, 807

Fiege, J., \& Henriksen, R.N. 1996, MNRAS, 281, 1038

Foster, P.N., Chevalier, R.A. 1993, ApJ 416, 303 (FC93)

Fuller, G.A., \& Myers, P.C. 1992, ApJ, 384, 523

Fuller, G.A., Lada, E.A., Masson, C.R., \& Myers, P.C. 1995, ApJ, 454, 862

Ghez, A.M., Neugebauer, G., \& Matthews, K. 1993, AJ, 106, 2005

Goldsmith, P.F., Langer, W.D. 1978, ApJ, 222, 881

Gómez, M., Hartmann, L., Kenyon, S.J., Hewett, R. 1993, AJ, 105, 1927

Goodman, A.A., Benson, P.J., Fuller, G.A., Myers, P.C. 1993, ApJ, 406, 528

Goodman, A.A., Barranco, J.A., Wilner, D.J., Heyer, M.H. 1998, ApJ, 504, 223

Greene, T.P., \& Lada C.J. 1996, AJ, 112, 2184

Greene, T. P., Wilking B. A., André P., Young E. T., Lada C. J. 1994, ApJ, 434, 614

Gregersen, E. M., Evans II, N. J., Zhou, S., \& Choi, M. 1997, ApJ, 484, 256

Gregersen, E.M., Evans, N.J., II, Mardones, D., \& Myers, P.C. 2000, ApJ, 533, 440

Henning, Th., \& Launhardt, R. 1998, A\&A, 338, 223

Henning, Th., Michel, B., Stognienko, R. 1995, P\&SS, 43, 1333

Henriksen, R.N. 1989, MNRAS, 240, 917

Henriksen, R.N., André, P., Bontemps, S. 1997, A\&A, 323, 549 (HAB97)

Hildebrand, R.H. 1983, QJRAS, 24, 267

Hogerheijde, M.R., \& Sandell, G. 2000, ApJ, 534, 880

Hogerheijde, M.R., van Dishoeck, E.F., Blake, G.A., \& van Langevelde, H.J. 1998, ApJ, 502,315

Jayawardhana, R., Hartmann, L., \& Calvet, N. 2001, ApJ, 548, 310

Jessop, N., \& Ward-Thompson, D. 2000, MNRAS, 311, 63

Jijina, J., Myers, P.C., Adams, F.C. 1999, ApJS, 125, 161

Johnstone, D., Wilson, C. D., Moriarty-Schieven, G., et al. 2000, ApJ, 545, 3274

Kane, B.D., \& Clemens D.P. 1997, AJ, 113, 1799

Kenyon, S.J., Hartmann, L.W. 1995, ApJS, 101, 117 
Kenyon, S.J., Calvet, N. \& Hartmann, L.W. 1993, ApJ, 414, 676

Kenyon, S.J., Brown, D.I., Tout, C.A., \& Berlind, P. 1998, AJ, 115, 2491

Klessen, R.S., \& Burkert, A. 2000, ApJS, 128, 287

Klessen, R.S., Heitsch, F., \& Mac Low, M.-M. 2000, ApJ, 535, 887

Königl, A., \& Pudritz, R.E. 2000, in Protostars and Planets IV, Eds. V. Mannings, A.P. Boss, \& S.S. Russell (Univ. of Arizona Press, Tucson), p. 759

Kramer, C., Stutzki, J., Rohrig, R., Corneliussen, U. 1998, A\&A, 329, 249

Kroupa, P., Tout, C. A., Gilmore, G. 1993, MNRAS, 262, 545

Lada, C.J. 1987, in Star Forming Regions (IAU Symp. 115), eds. M. Peimbert \& J. Jugaku (Reidel, Dordrecht), p. 1

Lada, C.J, \& Wilking, B.A. 1984, ApJ, 287, 610

Ladd, E.F., Fuller, G.A., Deane, J.R. 1998, ApJ, 495, 871

Ladd, E.F., Adams, F.C., Casey, S. et al. 1991, ApJ, 382, 555

Langer, W.D., van Dishoeck, E.F., Bergin, E.A. et al. 2000, in Protostars and Planets IV, Eds. V. Mannings, A.P. Boss, \& S.S. Russell (Univ. of Arizona Press, Tucson), p. 29 Larson, R.B. 1969, MNRAS, 145, 271

Larson, R.B. 1981, MNRAS, 194, 809

Larson, R.B. 1999, in Star Formation 1999, Ed. T. Nakamoto (Nobeyama Radio Observatory, Nobeyama), p. 336

Lay, O.P., Carlstrom, J.E., Hills, R.E. Phillips, T.G. 1994, ApJ, 434, L75

Lee, C.W, \& Myers, P.C. 1999, ApJS, 123, 233

Lee, C.W., Myers, P.C., \& Tafalla, M. 1999, ApJ, 526, 788

Lee, C.W., Myers, P.C., \& Tafalla, M. 2001, ApJS, in press (astro-ph/0105515)

Lehtinen, K., Lemke, D., Mattila, K., Haikala, L.K. 1998, A\&A, 333, 702

Li, Z.-Y. 1998, ApJ, 493, 230

Li, Z.-Y., Shu, F.H. 1996, ApJ, 472, 211

Li, Z.-Y., Shu, F.H. 1997, ApJ, 475, 237

Looney, L.W., Mundy, L.G., \& Welch, W.J. 2000, ApJ, 529, 477

Loren, R.B., Wootten, A., Wilking, B.A. 1990, ApJ, 365, 229

Lucas, P.W., \& Roche, P.F. 1997, MNRAS, 286, 895

Malbet, F. 2001, in The Origin of Stars and Planets: The VLT View, Eds. J. Alves \& M. McCaughrean (Springer-Verlag, Berlin), ESO Astrophysics Symposia, in press

Mardones, D., Myers, P. C., Tafalla, M., Wilner, D. J., Bachiller, R., Garay, G. 1997, ApJ, 489, 719

Masunaga, H., Inutsuka, S. 2000, ApJ, 531, 350

Mathis, J.S., Mezger, P.G., Panagia, N. 1983, A\&A, 128, 212

Matzner, C., \& McKee, C.F. 2000, ApJ, 545, 364

Menten, K.M., Serabyn, E., Güsten, R., \& Wilson, T.L. 1987, A\&A, 177, L57

Mizuno, A., Onishi, T., Hayashi, M. et al. 1994, Nature, 368, 719

Moriarty-Schieven G.H., Wannier P.G., Keene J., \& Tamura M. 1994, ApJ, 436, 800

Motte, F. 1998, PhD thesis, University of Paris XI

Motte, F., André, P. 2001, A\&A, 365, 440

Motte, F., André, P., Neri, R. 1998, A\&A, 336, 150 (MAN98)

Motte, F., André, P., Ward-Thompson, D., \& Bontemps, S. 2001, A\&A, 372, L41 (MAWB)

Mouschovias, T.Ch. 1991, in The Physics of Star Formation and Early Stellar Evolution, 
eds. C.J. Lada \& N. Kylafis (Kluwer, Dordrecht), p. 449

Mouschovias, T.Ch., \& Psaltis, D. 1995, ApJ, 444, L105

Mundy, L.G., Looney, L., Welch, J. 2000, in Protostars and Planets IV, Eds. V. Mannings, A.P. Boss, \& S.S. Russell (Univ. of Arizona Press, Tucson), p. 355

Myers, P.C. 1983, ApJ, 270, 105

Myers, P.C. 1998, ApJL, 496, L109

Myers, P.C. 2001, in From Darkness to Light, Eds. T. Montmerle \& P. André, ASP Conf. Ser., 243, p. 131

Myers, P.C., \& Fuller, G.A. 1992, ApJ, 396, 631

Myers, P.C., Evans, N.J.,II., \& Ohashi, N. 2000, in Protostars and Planets IV, Eds. V. Mannings, A.P. Boss, \& S.S. Russell (Univ. of Arizona Press, Tucson), p. 217

Myhill, E.A., \& Kaula, W.M. 1992, ApJ, 386, 578

Nakano, T. 1998, ApJ, 494, 587

Narayanan, G., Walker, C.K., \& Buckley, H.D. 1998, ApJ, 496, 292

Norman, C., \& Silk, J. 1980, ApJ, 238, 158

Ohashi, N. 1999, in Star Formation 1999, Ed. T. Nakamoto (Nobeyama Radio Observatory, Nobeyama), p. 129

Ohashi, N., Lee, S.W., Wilner, D.J., Hayashi, M. 1999, ApJL, 518, L41

Ossenkopf, V., \& Henning, Th. 1994, A\&A, 291, 943

Padoan, P., Nordlund, A., Rögnvaldsson, O.E., \& Goodman, A. 2001 in From Darkness to Light, Eds. T. Montmerle \& P. André, ASP Conf. Ser., 243, p. 279

Parker, N.D., Padman, R., \& Scott, P.F. 1991, MNRAS, 252, 442

Pilbratt, G.L., Cernicharo, J., Heras, A.M., \& Prusti, T. (eds.) 2001, The Promise of FIRST, ESA SP-460, in press

Pollack, J.B., Hollenbach, D., Beckwith, S., Simonelli, D.P., Roush, T., \& Fong, W. 1994, ApJ, 421, 615

Preibisch, Th., Ossenkopf, V., Yorke, H.W., \& Henning, Th. 1993, A\&A, 279, 577

Richer, J.S., Shepherd, D.S., Cabrit, S., Bachiller, R., \& Churchwell, E. 2000, in Protostars and Planets IV, Eds. V. Mannings, A.P. Boss, \& S.S. Russell (Univ. of Arizona Press, Tucson), p. 867

Rodríguez, L.F., D’Alessio, P., Wilner, D. J. et al. 1998, Nature, 395, 355

Safier, P.N., McKee, C.F., Stahler, S.W. 1997, ApJ, 485, 660

Saraceno, P., André, P., Ceccarelli, C., Griffin, M., \& Molinari, S. 1996 A\&A, 309, 827

Shirley, Y., Evans II, N.J., Rawlings, J.M.C., Gregersen, E.M. 2000, ApJS, 131, 249

Shu, F.H. 1977, ApJ, 214, 488

Shu, F.H., Adams, F.C., Lizano, S. 1987, ARA\&A, 25, 23

Shu, F.H., Najita, J., Galli, D., Ostriker, E., \& Lizano S. 1993, in Protostars and Planets III, Eds. E.H. Levy \& J.I. Lunine (Univ. of Arizona Press, Tucson), p. 3

Shu, F.H., Najita, J.R., Shang, H., \& Li, Z.-Y. 2000, in Protostars and Planets IV, Eds. V. Mannings, A.P. Boss, \& S.S. Russell (Univ. of Arizona Press, Tucson), p. 789

Siebenmorgen, R., \& Krügel, E. 2000, A\&A, 364, 625

Simon, M., Ghez, A.M., Leinert, Ch., Cassar, L. et al. 1995, ApJ, 443, 625

Stahler, S.W., \& Walter, F.M. 1993, in Protostars \& Planets III, Eds. E.H. Levy \& J.I. Lunine (Univ. of Arizona Press, Tucson), p. 405

Tafalla, M. 2001, in From Darkness to Light, Eds. T. Montmerle \& P. André, ASP Conf. Ser., 243, p. 103 
Tafalla, M., Mardones, D., Myers, P.C., Caselli, P., Bachiller, R., Benson, P.J. 1998, ApJ, 504,900

Terebey, S., Shu, F. H., \& Cassen, P. C., 1984, ApJ, 286, 529

Terebey, S., Van Buren, D., Hancock, T. et al. 2001 in From Darkness to Light, Eds. T. Montmerle \& P. André, ASP Conf. Ser., 243, p. 243

Testi, L., Sargent, A.I 1998, ApJL, 508, L91

Tomisaka, K. 2000, ApJL, 528, L41

van Dishoeck, E.F. \& Blake, G.A. 1998, ARA\&A, 36, 317

Vázquez-Semadeni, E., Ostriker, E.C., Passot, T., Gammie, C.F., \& Stone, J.M. 2000, in Protostars and Planets IV, eds. V. Mannings, A.P. Boss, \& S.S. Russell (Univ. of Arizona Press, Tucson), p. 3

Velusamy, T., Langer, W.D. 1998, Nature, 392, 685

Walker, C.K., Lada, C.J., Young, E.T., Maloney, P.R., Wilking, B.A. 1986, ApJ, 309, L47

Walmsley, M., Caselli, P., Zucconi, A., \& Galli, D. 2001, in The Origin of Stars and Planets: The VLT View, Eds. J. Alves \& M. McCaughrean (Springer-Verlag, Berlin), ESO Astrophysics Symposia, in press

Ward-Thompson, D., André, P., \& Kirk, J.M. 2001, MNRAS, in press

Ward-Thompson, D., Motte, F., André, P. 1999, MNRAS, 305, 143

Ward-Thompson, D., Scott, P.F., Hills, R.E., André, P. 1994, MNRAS, 268, 276

Ward-Thompson, D., Buckley, H.D., Greaves, J.S., Holland, W.S., \& André, P. 1996, MNRAS, 281, L53

Ward-Thompson, D., Kirk, J.M., Crutcher, R.M., Greaves, J.S., Holland, W.S., André, P. 2000, ApJL, 537, L135

Whitworth, A. 2001 in From Darkness to Light, Eds. T. Montmerle \& P. André, ASP Conf. Ser., 243, p. 171

Whitworth, A., Summers, D. 1985, MNRAS, 214, 1

Whitworth, A., \& Ward-Thompson, D. 2001, ApJ, 547, 317

Whitworth, A.P., Bhattal, A.S., Francis, N., \& Watkins, S.J. 1996, MNRAS, 283, 1061

Williams, J.P., Blitz, L., McKee, C. F. 2000, in Protostars \& Planets IV, Eds. V. Mannings, A. Boss, \& S. Russell (Univ. Arizona Press, Tucson), p. 97

Williams, J.P., Myers, P.C., Wilner, D.J., Di Francesco, J. 1999, ApJ, 513, L61

Wootten, A. 2001, Science with the Atacama Large Millimeter Array, ASP Conf. Ser., in press

Yorke, H. W., Bodenheimer, P., Laughlin, G. 1995, ApJ, 443, 199

Yun, J.L., Moreira, M.C., Torrelles, J.M., Afonso, J.M., Santos, N.C. 1996, AJ, 111, 841

Zhou, S., \& Evans, N.J. II, 1994, in Clouds, Cores, and Low Mass Stars, Eds. D.P. Clemens \& R. Barvainis, ASP Conf. Ser, 65, p. 183

Zhou, S., Evans, N.J., II, Wang, Y. 1996, ApJ, 466, 296

Zhou, S., Evans, N.J., II, Kömpe, C., \& Walmsley, C.M. 1993, ApJ, 404, 232

Zhou, S., Evans, N.J., II, Wang, Y., Peng, R., \& Lo, K.Y. 1994, ApJ. 433, 131 